\title{
Non-Gaussian Fixed Points of the Block Spin Transformation. Hierarchical Model Approximation
}

\author{
K. Gawędzki ${ }^{1 \star}$ and A. Kupiainen ${ }^{2}$ \\ 1 C.N.R.S., Institut des Hautes Etudes Scientifiques, F-91440 Bures-sur-Yvette, France \\ 2 Research Institute for Theoretical Physics, University of Helsinki, SF-00170 Helsinki, Finland
}

\begin{abstract}
With the use of analyticity techniques recently developed by the authors, the $\varepsilon$ - and $\frac{1}{N}$-expansion type arguments are turned into a rigorous control of the non-Gaussian fixed point of the hierarchical model renormalization group. The present approach should extend beyond the hierarchical approximation and result in mathematical theory of the critical point of statistical mechanics or quantum field theory in three dimensions for small $\varepsilon$ or large $N$.
\end{abstract}

\section{Introduction}

The present paper is the first step in the study of the critical point of the classical statistical mechanical systems with non-Gaussian long distance behaviour and of its scaling limit. As a presumed example of such a system one may consider a lattice model with the Gibbs state given formally by

$$
\frac{1}{Z} \exp \left[-\sum_{x} v\left(\phi_{x}\right)\right] d \mu_{G}(\boldsymbol{\phi})
$$

where the spin variables $\phi_{x} \in \mathbb{R}^{N}, x \in \mathbb{Z}^{d}, d \mu_{G}$ is the Gaussian measure with mean zero and covariance $G \equiv\left(G_{x y}\right)$ with $\left|G_{x y}\right| \sim|x-y|^{-\alpha}$ for large $|x-y|, \alpha<\frac{1}{2} d$, and where, for example, $v(\phi)=\frac{1}{2} m^{2} \boldsymbol{\phi}^{2}+\lambda\left(\boldsymbol{\phi}^{2}\right)^{2}$ with $m^{2} \in \mathbb{R}^{1}, \lambda>0$. The understanding of the behavior of (1) for the critical value of $\mathrm{m}^{2}$ (where the correlation length becomes infinite) is based on the renormalization group (RG) self-similarity idea $[1,11,14,15,20]$. Under RG transformations which integrate out successively the short range degrees of freedom, a critical system should go to a fixed point. In two situations: when $\varepsilon \equiv \frac{1}{2} d-\alpha$ or when $\frac{1}{N}$ are small, the RG transformation may be computed perturbatively as a formal power series in $\varepsilon$ or in closed form when

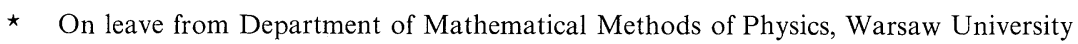


$N \rightarrow \infty[3,12-14,19,20]$. The relevant fixed point turns out to be non-Gaussian.

Despite a big success of the RG ideas due mainly to the existence of approximate computational schemes based on the $\varepsilon$ - and $\frac{1}{N}$-expansions, not many of the critical point properties have been established rigorously. The present paper constitutes a further development in the rigorous theory of the $R G$ which, as we hope, is a critical step towards filling this gap. We show how to control the corrections to the leading non-trivial order in $\varepsilon$ and $\frac{1}{N}$ of the $\mathrm{RG}$ transformation for a simplified, hierarchical model. In the case of small $\varepsilon$ our method provides the third proof of the convergence to a non-Gaussian fixed point, see [5-7] for the earlier ones. The large $N$ case seems to be new. What is more important, however, we are quite confident that our approach extends beyond the hierarchical approximation to (1), see $[9,10]$, where we treat a Gaussian fixed point case. Here we concentrate on the hierarchical model effective interactions, defering the study of the long distance behavior of the correlations to a later publication.

The model which we deal with is a version of the one introduced by Dyson $[8$, 4 , 7]. Let $\mathscr{A}(x)$ be a function on the $L \times \ldots \times L$ block around zero in $\mathbb{Z}^{d}, L$ being an even integer, which takes the value +1 on a half of the spins of the block and -1 on the other half. We put

$$
G_{x y}=\sum_{k=0}^{\infty} L^{-\alpha k} \delta_{x_{k+1} y_{k+1}} \mathscr{A}\left(x_{k}-L x_{k+1}\right) \mathscr{A}\left(y_{k}-L y_{k+1}\right),
$$

where $x_{k}$ denotes the integral part of $L^{-k} x$. It is easy to see that $\left|G_{x y}\right|=O\left(L^{-\alpha k_{0}}\right)$, where $k_{0}$ is the smallest integer such that $x_{k_{0}}=y_{k_{0}}$. Hence $G$ of (2) mimics the behavior $\left|G_{x y}\right| \sim|x-y|^{-\alpha}$. It is also easy to see that the kernel $G_{x y}$ defines a positive (but not strictly positive) operator.

The RG transformation which will be used to analyze (1) with $G$ given by (2) is the standard block spin transformation. Let us define the block spin field $\phi^{1}$ by

$$
\phi_{y}^{1}=L^{\frac{1}{2} \alpha-d} \sum_{x: x_{1}=y} \phi_{x} \equiv(C \phi)_{y} .
$$

The effective Gibbs state for the field $\phi^{1}$ is given (again formally) by

$$
\frac{1}{Z^{1}} D \boldsymbol{\phi}_{1} \int \delta\left(\boldsymbol{\phi}^{1}-C \boldsymbol{\phi}\right) \exp \left[-\sum_{x} \mathrm{v}\left(\boldsymbol{\phi}_{x}\right)\right] d \mu_{G}(\boldsymbol{\phi}) .
$$

Let us notice that we may realize $d \mu_{G}(\boldsymbol{\phi})$ as a product measure

$$
d \mu_{G}(\boldsymbol{\phi})=d \mu_{G}\left(\boldsymbol{\phi}^{\prime}\right) \times d \mu_{1}(\mathbf{Z}),
$$

provided we put

$$
\boldsymbol{\phi}_{x}=L^{-\frac{\alpha}{2}} \boldsymbol{\phi}_{x_{1}}^{\prime}+\mathscr{A}\left(x-L x_{1}\right) \mathbf{Z}_{x_{1}}
$$

This follows from the relation

$$
G_{x y}=L^{-\alpha} G_{x_{1} y_{1}}+\delta_{x_{1} y_{1}} \mathscr{A}\left(x-L x_{1}\right) \mathscr{A}\left(y-L y_{1}\right) .
$$


Inserting (5) and (6) to (4) we obtain

$$
\begin{aligned}
\text { (4) } & =\frac{1}{Z^{1}} d \mu_{G}\left(\boldsymbol{\phi}^{1}\right) \int \exp \left[-\sum_{x} \mathrm{v}\left(L^{-\frac{\alpha}{2}} \boldsymbol{\phi}_{x_{1}}^{1}+\mathscr{A}\left(x-L x_{1}\right) \mathbf{Z}_{x_{1}}\right)\right] d \mu_{1}(\mathbf{Z}) \\
& =\frac{1}{Z^{1}} \exp \left[-\sum_{x} v_{1}\left(\boldsymbol{\phi}_{x_{1}}^{1}\right)\right] d \mu_{G}\left(\boldsymbol{\phi}^{1}\right),
\end{aligned}
$$

where

$$
\begin{aligned}
\exp \left[-v_{1}(\boldsymbol{\varphi})\right]= & \operatorname{const} \int \exp \left[-\frac{1}{2} L^{d} v\left(L^{-\frac{\alpha}{2}} \boldsymbol{\varphi}+\mathbf{z}\right)\right. \\
& \left.-\frac{1}{2} L^{d} v\left(L^{-\frac{\alpha}{2}} \boldsymbol{\varphi}-\mathbf{z}\right)-\frac{1}{2} \mathbf{z}^{2}\right] d \mathbf{z}
\end{aligned}
$$

Due to the hierarchical character of $G$ the effective Gibbs measure for $\boldsymbol{\phi}^{1}$ is of the same form as the initial one for $\phi$. The block spin transformation reduces to the simple recursion (9) for the single spin potential $v$. This is the main simplification of the hierarchical model as compared to the more standard one with $G=\left(-\Delta_{\text {lattice }}\right)^{\frac{\alpha-d}{2}}$. For the sake of concreteness we shall limit ourselves to three dimensions. We shall also assume that $L$ is big enough (this is not essential). Two cases will be considered: $N=1,0<\varepsilon \equiv \frac{3}{2}-\alpha<\varepsilon_{0}(L)$ (Sects. 2 and 3) and $\alpha=1$, $N>N_{0}(L)$ (Sects. 4-6). The general strategy in both cases will be to find first a neighborhood of the approximate fixed point, preserved by the transformation (9) except for one relevant direction, and then to show that a distance between two successive iterations of (9) within this neighborhood shrinks at the critical point (i.e. when we are on the stable manifold of the fixed point). Somewhat unexpectedly in the large $N$ case, in the proofs it is enough to control perturbatively few terms of the Taylor expansion of $v$ around the minimum of the approximate fixed point and to estimate the remainders by means of the analyticity techniques. The analyticity techniques are also employed to estimate the contributions of large fluctuations to small $\boldsymbol{\phi}$ values of $v_{1}$ as given by (9). For these contributions the perturbative arguments break down. Instead we use a stability bound for $v$ (which carries on to $v_{1}$ ) together with a straightforward probability estimate for the Gaussian measure. All this is elementary although in some places tedious. We hope however, that technical estimates do not obscure the general idea to an extent which would render it non-readable.

\section{Small $\varepsilon$ Case. Invariant Neighborhood}

Denoting $\exp [-v]$ by $g$ we may rewrite (1.9) for $d=3, N=1$ and even $v$ vanishing at zero as

$$
g_{1}(\phi)=\int g\left(L^{-\frac{\alpha}{2}} \varphi+z\right)^{\frac{1}{2} L^{3}} g\left(L^{-\frac{\alpha}{2}} \varphi-z\right)^{\frac{1}{2} L^{3}} d v(z) / \int g(z)^{L^{3}} d v(z)
$$

where $d v(z) \equiv(2 \pi)^{-\frac{1}{2}} \exp \left[-\frac{1}{2} z^{2}\right] d z . \alpha=\frac{3}{2}-\varepsilon$ with small $\varepsilon>0$. Here $g \equiv 1$ is obviously a fixed point of (1). The linearized transformation around $g \equiv 1, \delta g \rightarrow \delta g^{\prime}$, 


$$
\delta g^{\prime}(\varphi)=L^{3} \int \delta g\left(L^{-\frac{\alpha}{2}} \varphi+z\right) d v(z)-L^{3} \int \delta g(z) d v(z)
$$

has (Hermite) polynomials of degree $2 n, n=1,2, \ldots$, as eigenvectors with eigenvalues $L^{3-\alpha n}$. For our choice of $\alpha, \varphi^{2}$ has the eigenvalue sizably bigger than 1 , the fourth order polynomial has the eigenvalue $1+O(\varepsilon)>1$ and the higher ones are irrelevant and have eigenvalues sizably smaller than 1 . We shall show that (1) has a non-trivial fixed point which has just bifurcated from the trivial one at $\varepsilon=0$ in the direction of the fourth order perturbation becoming relevant at this point $[5-7,16$, $19,20]$. The indication that this is what occurs can be obtained by taking $g=\exp \left[-\lambda \varphi^{4}\right]$ and computing the coefficient $\lambda_{1}$ at $\varphi^{4}$ in $-\log g_{1}(\varphi)$ to the second order in the perturbation expansion:

$$
\begin{aligned}
\lambda_{1} & =-\left.\frac{1}{4 !} \frac{d^{4}}{d \varphi^{4}}\right|_{\varphi=0} \log \int \exp \left[-\frac{1}{2} L^{3} \lambda\left(\left(L^{-\frac{\alpha}{2}} \varphi+z\right)^{4}+\left(L^{-\frac{\alpha}{2}} \varphi-z\right)^{4}\right)\right] d v(z) \\
& =L^{3-2 \alpha} \lambda-36 L^{6-2 \alpha} \lambda^{2}+O\left(\lambda^{3}\right) .
\end{aligned}
$$

Disregarding the $O\left(\lambda^{3}\right)$ corrections we obtain the non-trivial fixed point at

$$
\bar{\lambda}=\frac{1}{36} L^{-3}\left(1-L^{2 \alpha-3}\right) \text {. }
$$

The critical moment in our study of (1) is the choice of the functional space of $g$ 's and of its portion dominated by the non-trivial fixed point. Let us subtract the quadratic part of $\log g(\varphi)$ by introducing

$$
\tilde{g}(\varphi)=g(\varphi) \exp \left[a \varphi^{2}\right],
$$

with

$$
\tilde{g}^{\prime \prime}(0)=0 \text {. }
$$

We shall impose the following conditions on $\tilde{g}$ 's :

(a) $\tilde{g}(\varphi)$ is an even analytic function for $|\operatorname{Im} \varphi|<|\log \varepsilon|$, positive for real $\varphi$, $\tilde{g}(0)=1, \tilde{g}^{\prime \prime}(0)=0$.

(b) For $|\varphi|<|\log \varepsilon|$,

$$
\tilde{g}(\varphi)=\exp [-\tilde{v}(\varphi)]
$$

for analytic $\tilde{v}$, and

$$
\tilde{v}(\varphi)=\lambda \varphi^{4}+\tilde{\tilde{v}}(\varphi)
$$

where

$$
\tilde{\tilde{v}}(0)=\tilde{\tilde{v}}^{\prime \prime}(0)=\tilde{v}^{i v}(0)=0,
$$

and

$$
\varepsilon^{1 / 4}|\lambda-\bar{\lambda}|+\sup _{|\varphi|<|\log \varepsilon|}|\tilde{v}| \leqq \varepsilon^{7 / 4} .
$$

(c) For $|\varphi| \geqq|\log \varepsilon|$ and $|\operatorname{Im} \varphi|<L^{-\frac{\alpha}{2}}|\log \varepsilon|$,

$$
|g(\varphi)| \leqq \exp \left[-\frac{1}{4} \bar{\lambda}\left((\operatorname{Re} \varphi)^{4}+\kappa(\operatorname{Re} \varphi)^{2}\right)\right] .
$$


In the present section we shall show that our assumptions are invariant under (1) modulo a displacement in the relevant $\varphi^{2}$ direction.

Proposition 1. Let $\tilde{g}$ satisfy (a)-(c) and let $|a| \leqq 3 \varepsilon^{2 / 3}$. Then $\tilde{g}_{1}$ also satisfies (a)-(c) provided that $L>L_{0}, \varepsilon<\varepsilon_{0}(L)$ and $\kappa>\kappa_{0}(L)$.

Proof. Notice that (a) for $\tilde{g}_{1}$ follows easily as the integral of (1) converges absolutely uniformly in $\varphi$ by virtue of the bounds of (b) and (c) for $\tilde{g}$. The main contribution to $\tilde{g}_{1}(\varphi)$ comes from the integration over $|z|<\delta|\log \varepsilon|$ in (1) with small positive $\delta$. Let $\chi$ be the characteristic function of this region. Take $|\varphi|<L^{\frac{\alpha}{2}}(1-\delta)|\log \varepsilon|$ first. Define

$$
\begin{aligned}
g_{1 M}(\varphi)= & \int g\left(L^{-\frac{\alpha}{2}} \varphi+z\right)^{\frac{1}{2} L^{3}} g\left(L^{-\frac{\alpha}{2}}-z\right)^{\frac{1}{2} L^{3}} \chi(z) d v(z) / \int g(z)^{L^{3}} \chi(z) d v(z) \\
= & \exp \left[-L^{3-\alpha} a \varphi^{2}-L^{3-2 \alpha} \lambda \varphi^{4}\right] \int \exp \left[-L^{3} a z^{2}-6 L^{3-\alpha} \lambda \varphi^{2} z^{2}-L^{3} \lambda z^{4}\right. \\
& \left.-L^{3} \tilde{\tilde{w}}(\varphi, z)\right] \chi(z) d v(z) / \int \exp \left[-L^{3} a z^{2}-L^{3} \lambda z^{4}-L^{3} \tilde{\tilde{v}}(z)\right] \chi(z) d v(z),
\end{aligned}
$$

where

$$
\tilde{\tilde{w}}(\varphi, z) \equiv \frac{1}{2} \tilde{\tilde{v}}\left(L^{-\frac{\alpha}{2}} \varphi+z\right)+\frac{1}{2} \tilde{\tilde{v}}\left(L^{-\frac{\alpha}{2}} \varphi-z\right)
$$

Since $g_{1 M}(\varphi)=1+O\left(\varepsilon^{2 / 3}|\log \varepsilon|^{2}\right), g_{1 M}(\varphi)=\exp \left[-v_{1 M}(\varphi)\right]$ for a function $v_{1 M}$ analytic for $|\varphi|<L^{\frac{\alpha}{2}}(1-\delta)|\log \varepsilon|$, provided that $\varepsilon$ is small. The $v_{1 M}$ will be analyzed by perturbation expansion (with a remainder). With the use of identity

for

$$
f(1)=f(0)+f^{\prime}(0)+\frac{1}{2} f^{\prime \prime}(0)+\int_{0}^{1} d t\left(\frac{1}{2} t^{2}-t+\frac{1}{2}\right) f^{\prime \prime \prime}(t),
$$

$$
f(t)=\log \int \exp \left[-L^{3} a z^{2}-6 L^{3-\alpha} \lambda \varphi^{2} z^{2} t-L^{3} \lambda z^{4}-L^{3} \tilde{\tilde{w}}(\varphi, z)\right] \chi(z) d v(z),
$$

we may write

$$
\begin{aligned}
v_{1 M}(\varphi)= & L^{3-\alpha} a \varphi^{2}+L^{3-2 \alpha} \lambda \varphi^{4}-\left[||_{t=0}+\left.\frac{d}{d t}\right|_{t=0}+\left.\frac{1}{2} \frac{d^{2}}{d t^{2}}\right|_{t=0}\right. \\
& \left.+\int_{0}^{1} d t\left(\frac{1}{2} t^{2}-t+\frac{1}{2}\right) \frac{d^{3}}{d t^{3}}\right] \log \int \exp \left[-L^{3} a z^{2}-6 L^{3-\alpha} \lambda \varphi^{2} z^{2} t-L^{3} \lambda z^{4}\right. \\
& \left.-L^{3} \tilde{\tilde{w}}(\varphi, z)\right] \chi(z) d v(z)+\log \int \exp \left[-L^{3} a z^{2}-L^{3} \lambda z^{4}-L^{3} \tilde{\tilde{v}}(z)\right] \chi(z) d v(z) \\
= & L^{3-\alpha} a \varphi^{2}+L^{3-2 \alpha} \lambda \varphi^{4}+6 L^{\tilde{3}-\alpha}\left\langle z^{2}\right\rangle_{0} \lambda \varphi^{2}-18 L^{6-2 \alpha}\left\langle z^{2} ; z^{2}\right\rangle_{0}^{T} \lambda^{2} \varphi^{4} \\
& +216 L^{9-3 \alpha} \int_{0}^{1} d t\left(\frac{1}{2} t^{2}-t+\frac{1}{2}\right)\left\langle z^{2} ; z^{2} ; z^{2}\right\rangle_{t}^{T} \lambda^{3} \varphi^{6}-\log \int \exp \left[-L^{3} a z^{2}\right. \\
& \left.-L^{3} \lambda z^{4}-L^{3} \tilde{\tilde{w}}(\varphi, z)\right] \chi(z) d v(z) \\
& +\log \int \exp \left[-L^{3} a z^{2}-L^{3} \lambda z^{4}-L^{3} \tilde{\tilde{v}}(z)\right] \chi(z) d v(z),
\end{aligned}
$$

where

$$
\begin{aligned}
\langle-\rangle_{t} \equiv & \int-\exp \left[-L^{3} a z^{2}-6 L^{3-\alpha} \lambda \varphi^{2} z^{2} t-L^{3} \lambda z^{4}-L^{3} \tilde{\tilde{w}}(\varphi, z)\right] \\
& \cdot \chi(z) d v(z) \text { /normalization }
\end{aligned}
$$


and $\langle-; \ldots ;-\rangle_{t}^{T}$ denotes the truncated expectation. Decomposing [see (5) and (8)]

$$
v_{1 M}(\varphi)=a_{1 M} \varphi^{2}+\lambda_{1 M} \varphi^{4}+\tilde{\tilde{v}}_{1 M}(\varphi),
$$

we obtain from (16)

$$
\begin{aligned}
a_{1 M}= & L^{3-\alpha} a+\left.6 L^{3-\alpha}\left\langle z^{2}\right\rangle_{0}\right|_{\varphi=0} \lambda-\left.\frac{1}{2} \frac{d^{2}}{d \varphi^{2}}\right|_{\varphi=0} \log X, \\
\lambda_{1 M}= & L^{3-2 \alpha} \lambda-\left.18 L^{6-3 \alpha}\left\langle z^{2} ; z^{2}\right\rangle_{0}^{T}\right|_{\varphi=0} \lambda^{2}+\left.3 L^{3-\alpha} \frac{d^{2}}{d \varphi^{2}}\right|_{\varphi=0}\left\langle z^{2}\right\rangle_{0} \lambda \\
- & \left.\frac{1}{4 !} \frac{d^{4}}{d \varphi^{4}}\right|_{\varphi=0} \log X, \\
\tilde{v}_{1 M}(\varphi)= & 6 L^{3-\alpha}\left(\left\langle z^{2}\right\rangle_{0}-\left.\left\langle z^{2}\right\rangle_{0}\right|_{\varphi=0}-\left.\frac{1}{2} \varphi^{2} \frac{d^{2}}{d \varphi^{2}}\right|_{\varphi=0}\left\langle z^{2}\right\rangle_{0}\right) \lambda \varphi^{2} \\
& -18 L^{6-2 \alpha}\left(\left\langle z^{2} ; z^{2}\right\rangle_{0}^{T}-\left.\left\langle z^{2} ; z^{2}\right\rangle_{0}^{T}\right|_{\varphi=0}\right) \lambda^{2} \varphi^{4} \\
& +216 L^{9-3 \alpha} \int_{0}^{1} d t\left(\frac{1}{2} t^{2}-t+\frac{1}{2}\right)\left\langle z^{2} ; z^{2} ; z^{2}\right\rangle_{t}^{T} \lambda^{3} \varphi^{6} \\
& -\left(\log X-\left.\frac{1}{2} \varphi^{2} \frac{d^{2}}{d \varphi^{2}}\right|_{\varphi=0} \log X-\left.\frac{1}{4 !} \varphi^{4} \frac{d^{4}}{d \varphi^{4}}\right|_{\varphi=0} \log X\right),
\end{aligned}
$$

with

$$
\begin{aligned}
X \equiv & \int \exp \left[-L^{3} a z^{2}-L^{3} \lambda z^{4}-L^{3} \tilde{\tilde{w}}(\varphi, z)\right] \\
& \cdot \chi(z) d v(z) / \int \exp \left[-L^{3} a z^{2}-L^{3} \lambda z^{4}\right] \chi(z) d v(z) .
\end{aligned}
$$

First let us estimate $\left|\lambda_{1 M}-\bar{\lambda}\right|$. We shall do it step-wise.

$$
\begin{aligned}
\left|L^{3-2 \alpha} \lambda-36 L^{6-2 \alpha} \lambda^{2}-\bar{\lambda}\right| & =\left|L^{3-2 \alpha} \lambda-36 L^{6-2 \alpha} \lambda^{2}-L^{3-2 \alpha} \bar{\lambda}+36 L^{6-2 \alpha} \bar{\lambda}^{2}\right| \\
& \leqq \sup _{0<\theta<1}\left|L^{3-2 \alpha}-72 L^{6-2 \alpha}(\bar{\lambda}+\theta(\lambda-\bar{\lambda}))\right||\lambda-\bar{\lambda}| \\
& \leqq\left(\left|L^{3-2 \alpha}-72 L^{6-2 \alpha} \bar{\lambda}\right|+O\left(\varepsilon^{3 / 2}\right)\right)|\lambda-\bar{\lambda}| \\
& \leqq\left(1-\left(\log L^{2}\right) \varepsilon+O\left(\varepsilon^{3 / 2}\right)\right)|\lambda-\bar{\lambda}|
\end{aligned}
$$

where we have used the fact that $\bar{\lambda}$ is a fixed point of (3) and relations (4) and (10). Next

$$
\left|18 L^{6-2 \alpha}\left(2-\left.\left\langle z^{2} ; z^{2}\right\rangle_{0}^{T}\right|_{\varphi=0}\right) \lambda^{2}\right| \leqq O\left(\varepsilon^{8 / 3}\right),
$$

since $\lambda=O(\varepsilon)$ and $\left.\left\langle z^{2} ; z^{2}\right\rangle_{0}^{T}\right|_{\varphi=0}$ receives the main contribution from the perturbation in $a$. Similarly, using also the Cauchy estimates for derivatives of the analytic function, we obtain

$$
\left|3 L^{3-\alpha} \frac{d^{2}}{d \varphi^{2}}\right|_{\varphi=0}\left\langle z^{2}\right\rangle_{0} \lambda|\leqq O(\varepsilon) \sup | \tilde{v} \mid
$$


and

$$
\left|\frac{1}{4 !} \frac{d^{4}}{d \varphi^{4}}\right|_{\varphi=0} \log X|\leqq O(1) \sup | \tilde{\tilde{v}} \mid .
$$

Gathering of (23)-(26) gives

$$
\left|\lambda_{1 M}-\bar{\lambda}\right| \leqq\left(1-\frac{3}{2}(\log L) \varepsilon\right)|\lambda-\bar{\lambda}|+O(1) \sup |\tilde{v}|+O\left(\varepsilon^{8 / 3}\right) .
$$

Next we estimate $\sup _{|\varphi|<|\log \varepsilon|}\left|\tilde{\tilde{v}}_{1 M}\right|$.

$$
\begin{gathered}
\left|6 L^{3-2 \alpha}\left(\left\langle z^{2}\right\rangle_{0}-\left.\left\langle z^{2}\right\rangle_{0}\right|_{\varphi=0}-\left.\frac{1}{2} \varphi^{2} \frac{d^{2}}{d \varphi^{2}}\right|_{\varphi=0}\left\langle z^{2}\right\rangle_{0}\right) \lambda \varphi^{2}\right| \leqq O\left(\varepsilon|\log \varepsilon|^{2}\right) \sup |\tilde{v}| \\
\left|18 L^{6-2 \alpha}\left(\left\langle z^{2} ; z^{2}\right\rangle_{0}^{T}-\left.\left\langle z^{2} ; z^{2}\right\rangle_{0}^{T}\right|_{\varphi=0}\right) \lambda^{2} \varphi^{4}\right| \leqq O\left(\varepsilon^{2}|\log \varepsilon|^{4}\right) \sup |\tilde{v}| \\
\left|216 L^{9-3 \alpha} \int_{0}^{1} d t\left(\frac{1}{2} t^{2}-t+\frac{1}{2}\right)\left\langle z^{2} ; z^{2} ; z^{2}\right\rangle_{t}^{T} \lambda^{3} \varphi^{6}\right| \leqq O\left(\varepsilon^{3}|\log \varepsilon|^{6}\right)
\end{gathered}
$$

To estimate the last (and the main) term of (21), notice that for $|\varphi|<L^{\frac{\alpha}{2}}(1-\delta)|\log \varepsilon|$

$$
|X-1| \leqq L^{3} \exp \left[O\left(\varepsilon^{7 / 4}\right)\right] \sup _{|\varphi|<|\log \varepsilon|}|\tilde{\tilde{v}}|,
$$

and consequently $|\log X|$ satisfies the same bound.

Now for $|\varphi|<|\log \varepsilon|$, using the Cauchy estimates, we obtain

$$
\begin{aligned}
& \left|\log X-\frac{1}{2} \varphi^{2} \frac{d^{2}}{d \varphi^{2}}\right|_{\varphi=0} \log X-\left.\frac{1}{4 !} \varphi^{4} \frac{d^{4}}{d \varphi^{4}}\right|_{\varphi=0} \log X \mid \\
& \quad \leqq \sum_{k=3}^{\infty} \frac{1}{(2 k) !}|\varphi|^{2 k}\left|\frac{d^{2 k}}{d \varphi^{2 k}}\right|_{\varphi=0} \log X \mid \\
& \quad \leqq \sum_{k=3}^{\infty} \frac{L^{-\alpha k}}{(1-\delta)^{2 k}} \sup _{|\varphi|<L \frac{\alpha}{2}(1-\delta)|\log \varepsilon|}|\log X| \\
& \quad \leqq L^{3-3 \alpha}(1-\delta)^{-4}\left((1-\delta)^{2}-L^{-\alpha}\right)^{-1} \exp \left[O\left(\varepsilon^{7 / 4}\right)\right] \sup _{|\varphi|<|\log \varepsilon|}|\tilde{v}| \\
& \quad \leqq \frac{2}{3} \sup _{|\varphi|<|\log \varepsilon|}|\tilde{\tilde{v}}|
\end{aligned}
$$

provided that $L>L_{0}$. Inequalities (28)-(30) and (32) give

$$
\sup _{|\varphi|<|\log \varepsilon|}\left|\tilde{\tilde{v}}_{1 M}\right| \leqq \frac{3}{4} \sup _{|\varphi|<|\log \varepsilon|}|\tilde{\tilde{v}}|+O\left(\varepsilon^{3}|\log \varepsilon|^{6}\right) .
$$

Summarizing, (27), (33), and (10) yield

$$
\begin{aligned}
\varepsilon^{1 / 4}\left|\lambda_{1 M}-\bar{\lambda}\right|+\sup \left|\tilde{\tilde{v}}_{1 M}\right| \leqq & \left(1-\frac{3}{2}(\log L) \varepsilon\right)\left(\varepsilon^{1 / 4}|\lambda-\bar{\lambda}|+\sup |\tilde{\tilde{v}}|\right) \\
& +O\left(\varepsilon^{35 / 12}\right) \leqq(1-(\log L) \varepsilon) \varepsilon^{7 / 4}
\end{aligned}
$$

We are left with bounding the contributions to $g_{1}(\varphi)$ for $|\varphi|<|\log \varepsilon|$ from the integration over $|z| \geqq \delta|\log \varepsilon|$, disregarded so far. These should be damped by small 
$d v$-probability $\left(\sim \varepsilon^{O(|\log \varepsilon|)}\right)$ of such $z$. Indeed. From (1) and (12) we have

$$
\begin{aligned}
g_{1}(\varphi)= & g_{1 M}(\varphi)\left[1+\int g\left(L^{-\frac{\alpha}{2}} \varphi+z\right)^{\frac{1}{2} L^{3}} g\left(L^{-\frac{\alpha}{2}} \varphi-z\right)^{\frac{1}{2} L^{3}}\right. \\
& \left.\cdot \chi^{\perp}(z) d v(z) / \int g\left(L^{-\frac{\alpha}{2}} \varphi+z\right)^{\frac{1}{2} L^{3}} g\left(L^{-\frac{\alpha}{2}} \varphi-z\right)^{\frac{1}{2} L^{3}} \chi(z) d v(z)\right] \\
& \cdot\left[1+\int g(z)^{L^{3}} \chi^{\perp}(z) d v(z) / \int g(z)^{L^{3}} \chi(z) d v(z)\right]^{-1},
\end{aligned}
$$

where

$$
\chi^{\perp} \equiv 1-\chi
$$

Since by (a)-(c), $\left|\mathrm{g}\left(L^{-\frac{\alpha}{2}} \varphi \pm z\right)\right|<2$, say, for any $z$ and

$$
\left|g\left(L^{-\frac{\alpha}{2}} \varphi \pm z\right)-1\right| \leqq O\left(\varepsilon^{2 / 3}|\log \varepsilon|^{2}\right)
$$

if $\chi(z) \neq 0$ ( $\varepsilon$ small), we immediately obtain for the correction $v_{1 C} \equiv a_{1} \varphi^{2}+\tilde{v}_{1}-v_{1 M}$,

$$
\sup _{|\varphi|<|\log \varepsilon|}\left|v_{1 C}\right| \leqq \varepsilon^{O(|\log \varepsilon|)} .
$$

Taking into account the region $|z| \geqq \delta|\log \varepsilon|$ gives rise to corrections of infinite order in $\varepsilon$. Thus (b) for $\tilde{g}_{1}$ follows from (34).

We still have to show that the large field estimate of (c) holds for $\tilde{g}_{1}$. Let us take $\varphi$ with

$$
|\varphi| \geqq|\log \varepsilon| \quad \text { and } \quad|\operatorname{Im} \varphi|<L^{-\frac{\alpha}{2}}|\log \varepsilon| .
$$

We may rewrite (1) as

$$
\begin{aligned}
\tilde{g}_{1}(\varphi)= & \exp \left[\left(a_{1}-L^{3-\alpha} a\right) \varphi^{2}\right]\left(\int_{|z|<\frac{1}{2} L^{-\frac{\alpha}{2}}|\operatorname{Re} \varphi|}+\int_{|z| \geqq \frac{1}{2} L^{-\frac{\alpha}{2}}|\operatorname{Re} \varphi|}\right) \tilde{g}\left(L^{-\frac{\alpha}{2}} \varphi+z\right)^{\frac{1}{2} L^{3}} \\
& \cdot \tilde{g}\left(L^{-\frac{\alpha}{2}} \varphi-z\right)^{\frac{1}{2} L^{3}} \exp \left[-L^{3} a z^{2}\right] d v(z) / \int \tilde{g}(z)^{L^{3}} \exp \left[-L^{3} a z^{2}\right] d v(z) .
\end{aligned}
$$

Consider $|z|<\frac{1}{2} L^{-\frac{\alpha}{2}}|\operatorname{Re} \varphi|$ first. If $\left|L^{-\frac{\alpha}{2}} \varphi \pm z\right| \geqq|\log \varepsilon|$ then by (c)

$$
\left|\tilde{g}\left(L^{-\frac{\alpha}{2}} \varphi \pm z\right)\right| \leqq \exp \left[-\frac{1}{4} \bar{\lambda}\left(\left(L^{-\frac{\alpha}{2}} \operatorname{Re} \varphi \pm z\right)^{4}+\kappa\left(L^{-\frac{\alpha}{2}} \operatorname{Re} \varphi \pm z\right)^{2}\right)\right] .
$$

If $\left|L^{-\frac{\alpha}{2}} \varphi \pm z\right|<|\log \varepsilon|$ then, since

$$
\left|\operatorname{Re}\left(L^{-\frac{\alpha}{2}} \varphi \pm z\right)\right|>\frac{1}{2} L^{-\frac{\alpha}{2}}|\operatorname{Re} \varphi|>\frac{1}{2} L^{-\frac{\alpha}{2}}\left(1-L^{-\alpha}\right)^{1 / 2}|\log \varepsilon|
$$

and $\left|\operatorname{Im}\left(L^{-\frac{\alpha}{2}} \varphi \pm z\right)\right|<L^{-\alpha}|\log \varepsilon|, L^{-\frac{\alpha}{2}} \varphi \pm z$ still has a small argument $(L$ is big, $\varepsilon$ is 
small). Hence by virtue of (b),

$$
\begin{aligned}
\operatorname{Re}\left(\lambda\left(L^{-\frac{\alpha}{2}} \varphi \pm z\right)^{4}+\tilde{v}\left(L^{-\frac{\alpha}{2}} \varphi \pm z\right)\right) & \geqq \frac{1}{2} \bar{\lambda}\left(L^{-\frac{\alpha}{2}} \operatorname{Re} \varphi \pm z\right)^{4} \\
& \geqq \frac{1}{4} \bar{\lambda}\left(\left(L^{-\frac{\alpha}{2}} \operatorname{Re} \varphi \pm z\right)^{4}+\kappa\left(L^{-\frac{\alpha}{2}} \operatorname{Re} \varphi \pm z\right)^{2}\right)
\end{aligned}
$$

and (39) holds too. Now

$$
\begin{aligned}
& \left|\int_{|z|<\frac{1}{2} L^{-\frac{\alpha}{2}}|\operatorname{Re} \varphi|} \tilde{g}\left(L^{-\frac{\alpha}{2}} \varphi+z\right)^{\frac{1}{2} L^{3}} \tilde{g}\left(L^{-\frac{\alpha}{2}}-z\right)^{\frac{1}{2} L^{3}} \exp \left[-L^{3} a z^{2}\right] d v(z)\right| \\
& \leqq \exp \left[-\frac{1}{4} \bar{\lambda}\left(L^{3-2 \alpha}(\operatorname{Re} \varphi)^{4}+L^{3-\alpha} \kappa(\operatorname{Re} \varphi)^{2}\right)\right] \int \exp \left[-L^{3} a z^{2}\right] d v(z) \\
& \leqq \exp \left[-\frac{1}{4} \bar{\lambda}\left(L^{3-\alpha}-1\right) \kappa(\operatorname{Re} \varphi)^{2}\right] \exp \left[-\frac{1}{4} \bar{\lambda}\left((\operatorname{Re} \varphi)^{4}+\kappa(\operatorname{Re} \varphi)^{2}\right)\right] \\
& \quad \cdot \int \exp \left[-L^{3} a z^{2}\right] d v(z) .
\end{aligned}
$$

For $|z| \geqq \frac{1}{2} L^{-\alpha / 2}|\operatorname{Re} \varphi|$, either for + or for $-\operatorname{sign},\left|\operatorname{Re}\left(L^{-\alpha / 2} \varphi \pm z\right)\right| \geqq \frac{3}{2} L^{-\alpha / 2}|\operatorname{Re} \varphi|$ so that (39) holds. Thus

$$
\begin{aligned}
& \left|\int_{|z| \geqq \frac{1}{2} L^{-\frac{\alpha}{2}}|\operatorname{Re} \varphi|} \tilde{g}\left(L^{-\frac{\alpha}{2}} \varphi+z\right)^{\frac{1}{2} L^{3}} \tilde{g}\left(L^{-\frac{\alpha}{2}} \varphi-z\right)^{\frac{1}{2} L^{3}} \exp \left[-L^{3} a z^{2}\right] d v(z)\right| \\
& \leqq 2^{\frac{1}{2} L^{3}} \exp \left[-\frac{1}{8}\left(\frac{3}{2}\right)^{4} L^{3-2 \alpha} \bar{\lambda}(\operatorname{Re} \varphi)^{4}-\frac{1}{8}\left(\frac{3}{2}\right)^{2} L^{3-\alpha} \bar{\lambda} \kappa(\operatorname{Re} \varphi)^{2}\right] \varepsilon(|\log \varepsilon|) \\
& \leqq \varepsilon^{(|\log \varepsilon|)} \exp \left[-\frac{1}{4} \bar{\lambda}\left(L^{3-\alpha}-1\right) \kappa(\operatorname{Re} \varphi)^{2}\right] \exp \left[-\frac{1}{4} \bar{\lambda}\left((\operatorname{Re} \varphi)^{4}+\kappa(\operatorname{Re} \varphi)^{2}\right)\right] \\
& \quad \cdot \int \exp \left[-L^{3} a z^{2}\right] d v(z)
\end{aligned}
$$

and is a small correction to the bound (41).

As far as the $\exp \left[\left(a_{1}-L^{3-\alpha} a\right) \varphi^{2}\right]$ factor of (38) is concerned, we notice that, by virtue of (19) and (37),

$$
\left|a_{1}-L^{3-\alpha} a\right| \leqq O(\varepsilon)
$$

Finally, by (b) and (c),

$$
\int g(z)^{L^{3}} d v(z) \geqq \exp [-O(\varepsilon)] \int \exp \left[-L^{3} a z^{2}\right] d v(z) .
$$

Substituting (41)-(44) to (38) we obtain

$$
\begin{aligned}
\left|\tilde{g}_{1}(\varphi)\right| \leqq & \exp \left[O(\varepsilon)+O(\varepsilon)(\operatorname{Re} \varphi)^{2}-\frac{1}{4}\left[\bar{\lambda}\left(L^{3-\alpha}-1\right) \kappa(\operatorname{Re} \varphi)^{2}\right]\right. \\
& \cdot \exp \left[-\frac{1}{4} \bar{\lambda}\left((\operatorname{Re} \varphi)^{4}+\kappa(\operatorname{Re} \varphi)^{2}\right)\right] \leqq \exp \left[-\frac{1}{4} \bar{\lambda}\left((\operatorname{Re} \varphi)^{4}+\kappa(\operatorname{Re} \varphi)^{2}\right)\right]
\end{aligned}
$$

for $\kappa>\kappa_{0}(L)$. This demonstrates (c) for $\tilde{g}_{1}$ and completes Proof of Proposition 1.

\section{Small $\varepsilon$ Case. Contractive Properties of the RG Recursion}

Proposition 1 shows that the region of $g$ specified by (a)-(c) is invariant under the transformation (1), provided that $|a| \leqq 3 \varepsilon^{2 / 3}$. Unfortunately this assumption on the coefficient $a$ at the quadratic term of $-\log g$ is not stable under (1). As (2.43) shows, 
the main contribution to $a_{1}$ is $L^{3-\alpha} a$, in agreement with our analysis of the linearized RG transformation around $g \equiv 1: \varphi^{2}$ is a relevant perturbation. We shall however show that for one (critical) value of $a$ for each initial $\tilde{g}$, the assumptions of Proposition 1 for $a$ are stable under the iteration of (2.1). One also stays in the region specified by (a)-(c). Moreover, for the critical choice of $a$ the distance between subsequent iterates of (2.1) keeps shrinking, resulting in the convergence to a non-Gaussian fixed point.

Our strategy of finding the critical value of $a$ borrows from $[4,5]$. We shall restrict the admissible values of $a$ gradually from one iteration of (2.1) to the other so that finally the critical $a$ (corresponding to the critical value of the temperature) is chosen. Introduce

$$
b=a+6\left(1-L^{-\alpha}\right)^{-1}\left(1+2 L^{3} a\right)^{-1} \lambda .
$$

Taking $(b, \lambda)$ instead of $(a, \lambda)$ approximately diagonalizes, as we shall see, the RG transformation (2.1) restricted to (exp of) the subspace of the even fourth order polynomials.

Let us start from $\tilde{g}_{0}$ satisfying (a)-(c) and with $\left|a_{0}\right| \leqq \varepsilon^{2 / 3}$. By $g_{n}$ we shall denote the subsequent iterates of (2.1). Notice that, by virtue of (2.43), (2.20), and (2.37),

$$
\begin{aligned}
b_{1}-b_{0} & =a_{1}+6\left(1-L^{-\alpha}\right)^{-1}\left(1+2 L^{3} a_{1}\right)^{-1} \lambda_{1}-a_{0}-6\left(1-L^{-\alpha}\right)^{-1}\left(1+2 L^{3} a_{0}\right)^{-1} \lambda_{0} \\
& =L^{3-\alpha} a_{0}+6 L^{3-\alpha}\left(1-L^{-\alpha}\right)^{-1}\left(1+2 L^{3} a_{1}\right) \lambda_{0}-a_{0} \\
& -6\left(1-L^{-\alpha}\right)^{-1}\left(1+2 L^{3} a_{0}\right)^{-1} \lambda_{0}+O(\varepsilon)\left(a_{1}-a_{0}\right)+O\left(\varepsilon^{7 / 4}\right) \\
& =\left(L^{3-\alpha}-1\right) b_{0}+O(\varepsilon)\left(b_{1}-b_{0}\right)+O\left(\varepsilon^{7 / 4}\right) .
\end{aligned}
$$

From (2) it follows that there exists a closed interval $I_{0} C\left[-\varepsilon^{2 / 3}, \varepsilon^{2 / 3}\right]$ such that $b_{1}-b_{0}$ sweeps $\left[-\varepsilon^{5 / 3}, \varepsilon^{5 / 3}\right]$, say, if $a_{0}$ sweeps $I_{0}$. Below we list properties of $g_{n}$ 's to be proven by induction. In the light of the preceding discussion, it should be clear that $g_{0}$ and $g_{1}$ satisfy $\left(A_{0}\right)-\left(C_{0}\right)$. We use the notation $\Delta f_{n} \equiv f_{n+1}-f_{n}$ for any object indexed by $n$.

$\left(\mathrm{A}_{\mathrm{n}}\right)$. For $0 \leqq k \leqq n$ one can choose a closed interval $I_{k} \subset\left[-\varepsilon^{2 / 3}, \varepsilon^{2 / 3}\right], I_{k+1} \subset I_{k}$, such that $b_{k}$ sweeps $(1-\varepsilon)^{k}\left[\varepsilon^{5 / 3}, \varepsilon^{5 / 3}\right]$ as $a_{0}$ runs through $I_{k}$.

$\left(\mathrm{B}_{\mathrm{n}}\right)$. For $a_{0} \in I_{n}$,

$$
\varepsilon^{1 / 4}\left|\Delta \lambda_{n}\right|+\sup _{|\varphi|<|\log \varepsilon|}\left|\Delta \tilde{\tilde{v}}_{n}\right| \leqq 2(1-\varepsilon)^{n} \varepsilon^{7 / 4} .
$$

$\left(C_{\mathrm{n}}\right)$. For $a_{0} \in I_{n},|\varphi| \geqq|\log \varepsilon|$, and $|\operatorname{Im} \varphi|<L^{-\frac{\alpha}{2}}|\log \varepsilon|$

$$
\left|\Delta \tilde{g}_{n}(\varphi)\right| \leqq(1-\varepsilon)^{n}(\operatorname{Re} \varphi)^{6} \exp \left[-\frac{1}{4} \bar{\lambda}\left((\operatorname{Re} \varphi)^{4}+\kappa(\operatorname{Re} \varphi)^{2}\right)\right] .
$$

Notice that from $\left(\mathrm{A}_{\mathrm{n}}\right)$ it follows that for $a_{0} \in I_{n},\left|a_{k}\right| \leqq 3 \varepsilon^{2 / 3}$ for $0 \leqq k \leqq n+1$. Indeed. This holds for $k=0$. If moreover it does for $0 \leqq k \leqq l \leqq n$, then $g_{l}$ satisfies the assumptions of Proposition 1. Consequently, by Proposition 1 and its proof, $\left|\lambda_{l+1}\right|$ $\leqq O(\varepsilon)$ and $\left|a_{l+1}\right| \leqq O\left(\varepsilon^{2 / 3}\right)$. Now, by $(1)$,

$$
\left|a_{l+1}\right| \leqq\left|b_{l+1}-b_{0}\right|+\left|a_{0}\right|+O(\varepsilon) \leqq \sum_{k=0}^{l}(1-\varepsilon)^{k} \varepsilon^{5 / 3}+\varepsilon^{2 / 3}+O(\varepsilon) \leqq 3 \varepsilon^{2 / 3} .
$$

As a result we infer that the assumptions of Proposition 1 are satisfied for $g_{n+1}$ if $\left(A_{n}\right)$ holds. 
Proposition 2. Let $L>L_{0}, \varepsilon<\varepsilon_{0}(L), \kappa>\kappa_{0}(L)$. Then $\left(\mathrm{A}_{\mathrm{n}}\right)-\left(\mathrm{C}_{\mathrm{n}}\right)$ imply $\left(\mathrm{A}_{\mathrm{n}+1}\right)-\left(\mathrm{C}_{\mathrm{n}+1}\right)$. Proof. Consider small $\varphi,|\varphi|<L^{\frac{\alpha}{2}}(1-\delta)|\log \varepsilon|$, first. We have

$$
\begin{aligned}
\exp [ & \left.-\Delta a_{n+1} \varphi^{2}-\Delta \lambda_{n+1} \varphi^{4}-\Delta \tilde{\tilde{v}}_{n+1}(\varphi)\right] \equiv g_{n+2}(\varphi) / g_{n+1}(\varphi) \\
= & {\left[\int \operatorname { e x p } \left[-L^{3-\alpha} a_{n+1} \varphi^{2}-L^{3} a_{n+1} z^{2}-L^{3-2 \alpha} \lambda_{n+1} \varphi^{4}-6 L^{3-\alpha} \lambda_{n+1} \varphi^{2} z^{2}-L^{3} \lambda_{n+1} z^{4}\right.\right.} \\
& \left.\left.-L^{3} \tilde{\tilde{w}}_{n+1}(\varphi, z)\right] \chi(z) d v(z)+\int h_{n+1}(\varphi, z) \chi^{\perp}(z) d v(z)\right] \\
& \cdot\left[\int \operatorname { e x p } \left[-L^{3-\alpha} a_{n} \varphi^{2}-L^{3} a_{n} z^{2}-L^{3-2 \alpha} \lambda_{n} \varphi^{4}-6 L^{3-\alpha} \lambda_{n} \varphi^{2} z^{2}-L^{3} \lambda_{n} z^{4}\right.\right. \\
& \left.\left.-L^{3} \tilde{w}_{n}(\varphi, z)\right] \chi(z) d v(z)+\int h_{n}(\varphi, z) \chi^{\perp}(z) d v(z)\right]^{-1} \cdot \mathrm{const},
\end{aligned}
$$

where

$$
h_{n}(\varphi, z) \equiv g_{n}\left(L^{-\frac{\alpha}{2}} \varphi+z\right)^{\frac{1}{2} L^{3}} g_{n}\left(L^{-\frac{\alpha}{2}} \varphi-z\right)^{\frac{1}{2} L} \text {. }
$$

The main contribution to (6) comes again from the integration over small $z$. Dropping the terms with $\chi^{\perp}$ together with the overall $\varphi$-independent constant, omitting the subscript " $n$ " and replacing the " $n+1$ " one by the prime, we obtain the following expression for the main contribution to (6),

$$
\begin{aligned}
& \exp \left[\Delta c_{M}^{\prime}-\Delta a_{M}^{\prime} \varphi^{2}-\Delta \lambda_{M}^{\prime} \varphi^{4}+\Delta \tilde{\tilde{v}}_{M}^{\prime}(\varphi)\right]=\exp \left[-L^{3-\alpha} \Delta a \varphi^{2}-L^{3-2 \alpha} \Delta \lambda \varphi^{4}\right] \\
& \quad \cdot\left\langle\exp \left[-L^{3} \Delta a z^{2}-6 L^{3-\alpha} \Delta \lambda \varphi^{2} z^{2}-L^{3} \Delta \lambda z^{4}-L^{3} \Delta \tilde{\tilde{w}}(\varphi, z)\right]\right\rangle^{\chi},
\end{aligned}
$$

with $\Delta c_{M}^{\prime}$ being $\varphi$-independent and

$$
\begin{aligned}
\langle-\rangle^{\chi} \equiv & \int-\exp \left[-L^{3} a z^{2}-6 L^{3-\alpha} \lambda \varphi^{2} z^{2}-L^{3} \lambda z^{4}-L^{3} \tilde{\tilde{w}}(\varphi, z)\right] \\
& \cdot \chi(z) d v(z) / \text { normalization. }
\end{aligned}
$$

Notice that by $\left(\mathrm{A}_{\mathrm{n}}\right)-\left(\mathrm{C}_{\mathrm{n}}\right)$

$$
\left|L^{3} \Delta a z^{2}+6 L^{3-\alpha} \Delta \lambda \varphi^{2} z^{2}+L^{3} \Delta \lambda z^{4}+\Delta \tilde{\tilde{w}}(\varphi, z)\right| \leqq O\left((1-\varepsilon)^{n} \varepsilon^{3 / 2}|\log \varepsilon|^{4}\right) .
$$

Hence

$$
\begin{aligned}
\mid-\log & \left\langle\exp \left[-L^{3} \Delta a z^{2}-\ldots-L^{3} \Delta \tilde{\tilde{w}}(\varphi, z)\right]\right\rangle^{x} \\
& \left.-\left\langle L^{3} \Delta a z^{2}+\ldots+L^{3} \Delta \tilde{\tilde{w}}(\varphi, z)\right\rangle \mid \leqq O(1-\varepsilon)^{2 n} \varepsilon^{14 / 5}\right) .
\end{aligned}
$$

The $\left\langle L^{3} \Delta a z^{2}+\ldots+L^{3} \Delta \tilde{\tilde{w}}(\varphi, z)\right\rangle^{\chi}$ will again be computed by the perturbation expansion in the term $6 L^{3-\alpha} \lambda \varphi^{2} z^{2}$ of (9). Introducing $\langle-\rangle_{t}^{\chi}$, where $t$ multiplies this term, we may write

$$
\begin{aligned}
&\left\langle L^{3} \Delta a z^{2}+6 L^{3-\alpha} \Delta \lambda \varphi^{2} z^{2}+L^{3} \Delta \lambda z^{4}+L^{3} \Delta \tilde{w}(\varphi, z)\right\rangle^{\chi} \\
&= L^{3} \Delta a\left(\left\langle z^{2}\right\rangle_{0}^{\chi}-6 L^{3-\alpha} \lambda\left\langle z^{2} ; z^{2}\right\rangle_{0}^{\chi, T} \varphi^{2}+18 L^{6-2 \alpha} \lambda^{2}\left\langle z^{2} ; z^{2} ; z^{2}\right\rangle_{0}^{\chi, T} \varphi^{4}\right) \\
&-216 L^{9-3 \alpha} \lambda^{3} \Delta a \int_{0}^{1} d t\left(\frac{1}{2} t^{2}-t+\frac{1}{2}\right)\left\langle z^{2} ; z^{2} ; z^{2} ; z^{2}\right\rangle_{t}^{\chi, T} \varphi^{6} \\
&+6 L^{3-\alpha} \Delta \lambda\left(\left\langle z^{2}\right\rangle_{0}^{\chi}-6 L^{3-\alpha} \lambda\left\langle z^{2} ; z^{2}\right\rangle_{0}^{\chi, T} \varphi^{2}\right) \varphi^{2} \\
&+216 L^{9-3 \alpha} \Delta \lambda \lambda^{2} \int_{0}^{1} d t(1-t)\left\langle z^{2} ; z^{2}\right\rangle_{t}^{\chi, T} \varphi^{6} \\
&+L^{3} \Delta \lambda\left(\left\langle z^{4}\right\rangle_{0}^{\chi}-6 L^{3-\alpha} \lambda\left\langle z^{4} ; z^{2}\right\rangle_{0}^{\chi, T} \varphi^{2}+18 L^{6-2 \alpha} \lambda^{2}\left\langle z^{4} ; z^{2} ; z^{2}\right\rangle_{0}^{\chi, T} \varphi^{4}\right) \\
&-216 L^{9-3 \alpha} \lambda^{3} \Delta \lambda \int_{0}^{1} d t\left(\frac{1}{2} t^{2}-t+\frac{1}{2}\right)\left\langle z^{4} ; z^{2} ; z^{2} ; z^{2}\right\rangle_{0}^{\chi, T} \varphi^{6}+L^{3}\langle\tilde{w}(\varphi, z)\rangle^{\chi} .
\end{aligned}
$$


This tedious decomposition, together with (8) and (11), results in the estimates

$$
\begin{aligned}
& \left|\Delta c_{M}^{\prime}\right| \leqq O(|\Delta a|)+O(|\Delta \lambda|)+O(\sup |\Delta \tilde{\tilde{v}}|)+O\left((1-\varepsilon)^{2 n} \varepsilon^{14 / 5}\right) \leqq O\left((1-\varepsilon)^{n} \varepsilon^{3 / 2}\right), \\
& \left|\Delta a_{M}^{\prime}-L^{3-\alpha} \Delta a-6 L^{3-\alpha}\left(1+2 L^{3} a\right)^{-1} \Delta \lambda\right| \leqq O(\varepsilon)|\Delta \lambda|+O(1) \sup |\Delta \tilde{v}|+O(\varepsilon)|\Delta a| \\
& +O\left((1-\varepsilon)^{2 n} \varepsilon^{14 / 5}\right) \leqq O\left((1-\varepsilon)^{n} \varepsilon^{7 / 4}\right), \\
& \left|\Delta \lambda_{M}^{\prime}-L^{3-2 \alpha} \Delta \lambda+72 L^{6-2 \alpha} \bar{\lambda} \Delta \lambda\right| \leqq O\left(\varepsilon^{3 / 2}\right)|\Delta \lambda|+O(1) \sup |\Delta \tilde{\tilde{v}}| \\
& +O\left(\varepsilon^{7 / 4}\right)|\Delta a|+O\left((1-\varepsilon)^{2 n} \varepsilon^{14 / 5}\right) \\
& \left|\Delta \tilde{\tilde{v}}_{M}^{\prime}\right| \leqq O\left(\varepsilon^{7 / 4}\right)|\Delta \lambda|+\frac{3}{4} \sup |\Delta \tilde{\tilde{v}}|+O\left(\varepsilon^{7 / 4}\right)|\Delta a| \\
& +O\left((1-\varepsilon)^{2 n} \varepsilon^{14 / 5}\right) \text { for }|\varphi|<|\log \varepsilon| .
\end{aligned}
$$

From (3), (15), and (16) it follows that

$$
\varepsilon^{1 / 4}\left|\Delta \lambda_{M}^{\prime}\right|+\sup _{|\varphi|<|\log \varepsilon|}\left|\Delta \tilde{\tilde{v}}_{M}^{\prime}\right| \leqq 2(1-(\log L) \varepsilon)(1-\varepsilon)^{n} \varepsilon^{7 / 4} .
$$

We still have to estimate the errors coming from the large $z$ terms of (6) containing $\chi^{\perp}$. We must show not only that they are small for small $\varepsilon$ but also that they go down with $n$ at least as $(1-\varepsilon)^{n}$. By virtue of (6) and (8), we have

$$
\begin{aligned}
\exp \left[-\Delta a^{\prime} \varphi^{2}+\Delta \lambda^{\prime} \varphi^{4}+\Delta \tilde{\tilde{v}}^{\prime}(\varphi)\right]=\exp \left[-\Delta c_{M}^{\prime}-\Delta a_{M}^{\prime} \varphi^{2}-\Delta \lambda_{M}^{\prime} \varphi^{4}-\Delta \tilde{\tilde{v}}_{M}^{\prime}(\varphi)\right] \\
\quad \cdot\left\{1+\exp \left[\Delta c_{M}^{\prime}+\Delta a_{M}^{\prime} \varphi^{2}+\Delta \lambda_{M}^{\prime} \varphi^{4}+\Delta \tilde{\tilde{v}}_{M}^{\prime}(\varphi)\right] \int h^{\prime}(\varphi, z) \chi^{\perp}(z) d v(z) / \int h(\varphi, z) \chi(z) d v(z)\right. \\
\quad \cdot\left\{1+\int h(\varphi, z) \chi^{\perp}(z) d v(z) / \int h(\varphi, z) \chi(z) d v(z)\right\}^{-1} \cdot \text { const. }
\end{aligned}
$$

Notice that for $|\varphi|<|\log \varepsilon|$, by (13)-(16),

$$
\left|\exp \left[\Delta c_{M}^{\prime}+\Delta a_{M}^{\prime} \varphi^{2}+\Delta \lambda_{M}^{\prime} \varphi^{4}+\Delta \tilde{v}_{M}^{\prime}(\varphi)\right]-1\right| \leqq O\left((1-\varepsilon)^{n}\right),
$$

and by $\left(\mathrm{A}_{\mathrm{n}}\right)-\left(\mathrm{C}_{\mathrm{n}}\right)$

$\left|\left[\int h^{\prime}(\varphi, z) \chi^{\perp}(z) d v(z)-\int h(\varphi, z) \chi^{\perp}(z) d v(z)\right] / \int h(\varphi, z) \chi(z) d v(z)\right| \leqq(1-\varepsilon)^{n} \varepsilon^{o(|\log \varepsilon|)},(20)$

$$
\left|\int h(\varphi, z) \chi^{\perp}(z) d v(z) / \int h(\varphi, z) \chi(z) d v(z)\right| \leqq \varepsilon^{O(|\log \varepsilon|)} .
$$

Expressions (18)-(21) imply that

$$
\begin{gathered}
\left|\Delta a^{\prime}-\Delta a_{M}^{\prime}\right| \leqq(1-\varepsilon)^{n} \varepsilon^{O(|\log \varepsilon|)}, \\
\left|\Delta \lambda^{\prime}-\Delta \lambda_{M}^{\prime}\right| \leqq(1-\varepsilon)^{n} \varepsilon^{O(|\log \varepsilon|)}, \\
\sup _{|\varphi|<|\log \varepsilon|}\left|\Delta \tilde{\tilde{\mathrm{V}}}^{\prime}-\Delta \tilde{\mathrm{v}}_{1}^{\prime}\right| \leqq(1-\varepsilon)^{n} \varepsilon^{O(|\log \varepsilon|)} .
\end{gathered}
$$

Inequality (17) and (22)-(24) prove $\left(B_{n+1}\right)$.

To show $\left(A_{n+1}\right)$, notice that by (1), (3), (14), (15), (22), and (23),

$$
\begin{aligned}
\Delta b^{\prime} \equiv & \Delta b_{n+1}=a_{n+2}+6\left(1-L^{-\alpha}\right)^{-1}\left(1+2 L^{3} a_{n+2}\right)^{-1} \lambda_{n+2}-a_{n+1} \\
& -6\left(1-L^{-\alpha}\right)^{-1}\left(1+2 L^{3} a_{n+1}\right)^{-1} \lambda_{n+1}=\Delta a^{\prime}+6\left(1-L^{-\alpha}\right)^{-1}\left(1+2 L^{3} a\right)^{-1} \Delta \lambda^{\prime} \\
& +O\left((1-\varepsilon)^{n} \varepsilon^{5 / 2}\right)=L^{3-\alpha}\left(\Delta a+6\left(1-L^{-\alpha}\right)^{-1}\left(1+2 L^{3} a\right)^{-1} \Delta \lambda\right) \\
& +O\left((1-\varepsilon)^{n} \varepsilon^{7 / 4}\right)=L^{3-\alpha} \Delta b+O\left((1-\varepsilon)^{n} \varepsilon^{7 / 4}\right) .
\end{aligned}
$$


Since for $a_{0}$ running through $I_{n} C\left[-\varepsilon^{2 / 3}, \varepsilon^{2 / 3}\right], \Delta b$ sweeps $(1-\varepsilon)^{n}\left[-\varepsilon^{5 / 3}, \varepsilon^{5 / 3}\right]$ and $L^{3-\alpha}$ is sizably bigger than 1 , there exists $I_{n+1} \subset I_{n}$ such that $\Delta b_{n+1}$ sweeps $(1-\varepsilon)^{n+1}\left[-\varepsilon^{5 / 3}, \varepsilon^{5 / 3}\right]$ when $a$ runs through $I_{n+1}$. This proves $\left(A_{n+1}\right)$.

We are left with showing $\left(\mathrm{C}_{\mathrm{n}}\right)$. Let $|\varphi| \geqq|\log \varepsilon|$ and $|\operatorname{Im} \varphi|<L^{-\frac{\alpha}{2}}|\log \varepsilon|$. We have

$$
\begin{aligned}
\left|\tilde{g}_{n+2}(\varphi)-\tilde{g}_{n+1}(\varphi)\right| \leqq & \exp \left[\left(a_{n+2}-L^{3-\alpha} a_{n}\right) \varphi^{2}\right] \int \tilde{h}_{n+1}(\varphi, z) \\
& \cdot \exp \left[-L^{3} a_{n+1} z^{2}\right] d v(z) / \int \tilde{g}_{n+1}(z)^{L^{3}} \exp \left[-L^{3} a_{n+1} z^{2}\right] d v(z) \\
& -\exp \left[\left(a_{n+1}-L^{3-\alpha} a_{n}\right) \varphi^{2}\right] \int \tilde{h}_{n}(\varphi, z) \\
& \cdot \exp \left[-L^{3} a_{n} z^{2}\right] d v(z) / \int \tilde{g}_{n}(z)^{L^{3}} \exp \left[-L^{3} a_{n} z^{2}\right] d v(z) \mid
\end{aligned}
$$

where we have denoted

$$
\tilde{h}_{n}(\varphi, z) \equiv \tilde{g}_{n}\left(L^{-\frac{\alpha}{2}} \varphi+z\right)^{\frac{1}{2} L^{3}} \tilde{g}_{n}\left(L^{-\frac{\alpha}{2}} \varphi-z\right)^{\frac{1}{2} L^{3}} .
$$

Using (2.43), (14), (22), $\left(\mathrm{A}_{\mathrm{n}}\right)-\left(\mathrm{C}_{\mathrm{n}}\right)$, and (b) and (c) for $\tilde{g}_{n}$ and $\tilde{g}_{n+1}$, we obtain easily

$$
\begin{aligned}
& \mid \exp \left[\left(a_{n+2}-L^{3-\alpha} a_{n+1}\right) \varphi^{2}\right] / \int \tilde{g}_{n+1}(z)^{L^{3}} \exp \left[-L^{3} a_{n+1} z^{2}\right] d v(z) \\
& \quad-\exp \left[\left(a_{n+1}-L^{3-\alpha} a_{n}\right) \varphi^{2}\right] / \int \tilde{g}_{n}(z)^{L^{3}} \exp \left[-L^{3} a_{n} z^{2}\right] d v(z) \mid \\
& \leqq O\left((1-\varepsilon)^{n} \varepsilon^{3 / 2}|\log \varepsilon|^{2}\right)(\operatorname{Re} \varphi)^{2} \exp \left[O(\varepsilon)(\operatorname{Re} \varphi)^{2}\right] .
\end{aligned}
$$

Mimicking the steps (2.38)-(2.45) we show now with the use of (28) that

$$
\begin{aligned}
\mid \exp & {\left[\left(a_{n+2}-L^{3-\alpha} a_{n+1}\right) \varphi^{2}\right] \int \tilde{h}_{n+1}(\varphi, z) \exp \left[-L^{3} a_{n+1} z^{2}\right] d v(z) / \int \tilde{g}_{n+1}(z)^{L^{3}} } \\
& \cdot \exp \left[-L^{3} a_{n+1} z^{2}\right] d v(z)-\exp \left[\left(a_{n+1}-L^{3-\alpha} a_{n}\right) \varphi^{2}\right] \int \tilde{h}_{n+1}(\varphi, z) \\
& \cdot \exp \left[-L^{3} a_{n} z^{2}\right] d v(z) / \int \tilde{g}_{n}(z)^{L^{3}} \exp \left[-L^{3} a_{n} z^{2}\right] d v(z) \mid \\
\leqq & O\left((1-\varepsilon)^{n} \varepsilon^{3 / 2}|\log \varepsilon|^{2}\right)(\operatorname{Re} \varphi)^{2} \exp \left[-\frac{1}{4} \bar{\lambda}\left((\operatorname{Re} \varphi)^{4}+\kappa(\operatorname{Re} \varphi)^{2}\right)\right.
\end{aligned}
$$

Hence we are left with bounding

$$
\begin{aligned}
& \left|\exp \left[\left(a_{n+1}-L^{3-\alpha} a_{n}\right) \varphi^{2}\right] \int\left(\tilde{h}_{n+1}(\varphi, z)-\tilde{h}_{n}(\varphi, z)\right) \exp \left[-L^{3} a_{n} z^{2}\right] d v(z)\right| \\
& \leqq \exp \left[O(\varepsilon)(\operatorname{Re} \varphi)^{2}\right] L^{3} \int\left|\tilde{g}_{n+1}\left(L^{-\frac{\alpha}{2}} \varphi+z\right)-\tilde{g}_{n}\left(L^{-\frac{\alpha}{2}} \varphi-z\right)\right| \\
& \quad \cdot\left|\tilde{g}_{\#}\left(L^{-\frac{\alpha}{2}} \varphi+z\right)\right|^{\frac{1}{2} L^{3}-1}\left|\tilde{g}_{\#}\left(L^{-\frac{\alpha}{2}} \varphi-z\right)\right|^{\frac{1}{2} L^{3}} \exp \left[-L^{3} a_{n} z^{2}\right] d v(z),
\end{aligned}
$$

where

$$
\left|\tilde{g}_{\#}\right|=\max \left(\left|\tilde{g}_{n}\right|,\left|\tilde{g}_{n+1}\right|\right) \text {. }
$$

The right hand side of (30) is again estimated by mimicking the steps $(2.38)-(2.45)$ with the use of $\left(B_{n}\right)$ and $\left(C_{n}\right)$. The point is that the coefficient at $(\operatorname{Re} \varphi)^{6}$ in (4) goes down from $(1-\varepsilon)^{n}$ to $L^{3-3 \alpha}(1-\varepsilon)^{n}$. This way the term on the right hand side of (30) may be bounded by

$$
\left(1-\frac{1}{2}\left(1-L^{3-3 \alpha}\right)\right)(1-\varepsilon)^{n}(\operatorname{Re} \varphi)^{6} \exp \left[-\frac{1}{4} \bar{\lambda}\left((\operatorname{Re} \varphi)^{4}+\kappa(\operatorname{Re} \varphi)^{2}\right)\right] .
$$

Expressions (26), (29), (30), and (32) produce $\left(\mathrm{C}_{\mathrm{n}+1}\right)$. 
From the fact that $\left(\mathrm{A}_{\mathrm{n}}\right)-\left(\mathrm{C}_{\mathrm{n}}\right)$ hold for any $n$ it follows immediately that for $a_{0} \in \cap I_{k}, g_{n}$ converge uniformly for

$$
|\operatorname{Im} \varphi|<L^{-\frac{\alpha}{2}}|\log \varepsilon|
$$

to a fixed point $g_{\infty}$ of (2.1). That the uniform convergence holds for $|\operatorname{Im} \varphi|<|\log \varepsilon|$ follows easily from this and (2.1) to know $g_{n+1}(\varphi)$ for $\varphi$ with $|\operatorname{Im} \varphi|<|\log \varepsilon|$ we have to know $g_{n}(\varphi)$ only for $\varphi$ with $|\operatorname{Im} \varphi|<L^{-\frac{\alpha}{2}}|\log \varepsilon|$. Actually, subsequent $g_{n}$ are analytic for $|\operatorname{Im} \varphi|<L^{(n-1) \alpha / 2}|\log \varepsilon|$ and $g_{\infty}$ is an entire function.

This way we have obtained the following:

Theorem 1. Suppose that $L>L_{0}$. Given $\tilde{g}_{0}$ satisfying (a)-(c) of Sect. 2 with $\varepsilon<\varepsilon_{0}(L)$ and $\kappa>\kappa_{0}(L)$, there exists $a_{0},\left|a_{0}\right| \leqq \varepsilon^{2 / 3}$, such that the iterates of $(2.1), g_{n}(\varphi)$ $=\tilde{g}_{n}(\varphi) \exp \left[-a_{n} \varphi^{2}\right]$, converge to a fixed point of $(2.1), g_{\infty}$, uniformly for $|\operatorname{Im} \varphi|$ $<|\log \varepsilon|$. Moreover $\tilde{g}_{\infty}$ satisfies (a)-(c) and $\left|a_{\infty}\right| \leqq 3 \varepsilon^{2 / 3}$ (so in particular $g_{\infty}$ is nonGaussian).

\section{4. $N=\infty$ Fixed Point}

Now we shall consider the many component hierarchical model with $O(N)$-invariant single spin distributions $g\left(\varphi^{2} / N\right)$. Taking $\varphi=N^{1 / 2}(\varphi, 0, \ldots, 0)$, $\mathbf{z}=N^{1 / 2}\left(s, \zeta_{1}, \ldots, \zeta_{N-1}\right), \zeta_{1}^{2}+\ldots+\zeta_{N-1}^{2}=u$, we may rewrite (1.9) for $d=3$ and $\alpha=1$ as

$$
\begin{aligned}
g_{1}\left(\varphi^{2}\right)= & \text { const } \int_{-\infty}^{\infty} d s \int_{0}^{\infty} d u g\left(L^{-1} \varphi^{2}+2 L^{-1 / 2} \varphi s+s^{2}+u\right)^{\frac{1}{2} L^{3}} \\
& \cdot g\left(L^{-1} \varphi^{2}-2 L^{-1 / 2} \varphi s+s^{2}+u\right)^{\frac{1}{2} L^{3}} \\
& \cdot \exp \left[-\frac{N}{2}\left(s^{2}+u-\log u\right)-\frac{3}{2} \log u\right] .
\end{aligned}
$$

Putting

$$
g_{1}\left(\varphi^{2}\right)=\exp \left[-\frac{N}{2} v_{(1)}\left(\varphi^{2}\right)\right]
$$

we may compute (1) in the limit $N \rightarrow \infty$ by the steepest decent method. This was done for the first time by Ma [12-14]. Our discussion of the large $N$ limit will follow in main lines his original papers. Equations (1) and (2) give

$$
v_{1}\left(\varphi^{2}\right) \underset{N=\infty}{=} \inf _{s, u}\left[\frac{1}{2} L^{3} v\left(\psi^{+}\right)+\frac{1}{2} L^{3} v\left(\psi^{-}\right)+s^{2}+u-\log u\right]+\text { const },
$$

where

$$
\psi_{ \pm} \equiv L^{-1} \varphi^{2} \pm 2 L^{-1 / 2} \varphi s+s^{2}+u \text {. }
$$

If $v$ goes to $+\infty$ when $\varphi^{2}$ tends to $+\infty$, we obtain

$$
v_{1}\left(\varphi^{2}\right)_{N=\infty}=\frac{1}{2} L^{3} v\left(\psi_{0}\right)+\frac{1}{2} L^{3} v\left(\psi_{0}^{-}\right)+s_{0}^{2}+u_{0}-\log u_{0}+\text { const }
$$


where

and

$$
\left.\psi_{0}^{ \pm} \equiv \psi^{ \pm}\right|_{s_{0}, u_{0}}
$$

$$
\begin{gathered}
L^{3}\left[v^{\prime}\left(\psi_{0}\right)\left(L^{-1 / 2} \varphi+s_{0}\right)+v^{\prime}\left(\psi_{0}^{-}\right)\left(-L^{-1 / 2} \varphi+s_{0}\right)\right]+2 s_{0}=0, \\
\frac{1}{2} L^{3} v^{\prime}\left(\psi_{0}^{+}\right)+\frac{1}{2} L^{3} v^{\prime}\left(\psi_{0}^{-}\right)+1-\frac{1}{u_{0}}=0 .
\end{gathered}
$$

Here $s_{0}=0$ is a solution of (7), in fact the one relevant for the fixed point, as we shall see. Hence

$$
v_{1}\left(\varphi^{2}\right) \underset{N=\infty}{=} L^{3} v\left(L^{-1} \varphi^{2}+u_{0}\right)+u_{0}-\log u_{0}+\text { const },
$$

or, taking its derivative,

$$
v_{1}^{\prime}\left(\varphi^{2}\right) \underset{N=\infty}{=} L^{2} v^{\prime}\left(L^{-1} \varphi^{2}+u_{0}\right)
$$

where

$$
L^{3} v^{\prime}\left(L^{-1} \varphi^{2}+u_{0}\right)+1-\frac{1}{u_{0}}=0 .
$$

Denoting by $t, t_{1}$ the functions inverse to $v^{\prime}, v_{1}^{\prime}$ (assuming they exist) and setting $L^{2} v^{\prime}\left(L^{-1} \varphi^{2}+u_{0}\right)=\tau$, we may rewrite (10) and (11) as a single relation

$$
t_{1}(\tau)=L t\left(L^{-2} \tau\right)-L(1+L \tau)^{-1}
$$

For the fixed point $t$ this becomes

$$
t_{\infty}(\tau)=L t_{\infty}\left(L^{-2} \tau\right)-L(1+L \tau)^{-1} .
$$

Notice that $t_{\infty}(0)=L(L-1)^{-1} \equiv \varphi_{0}^{2}$. Here $\varphi_{0}^{2}$ is the extremum of the $N=\infty$ fixed point $v$. We may rewrite $(13)$ as

$$
\left[(1-M)\left(t_{\infty}-t_{\infty}(0)\right)\right](\tau)=L^{2} \tau(1+L \tau)^{-1},
$$

where

$$
M f(\tau) \equiv L f\left(L^{-2} \tau\right)
$$

Formally at least

$$
\begin{aligned}
t_{\infty}(\tau) & =t_{\infty}(0)+\sum_{n=0}^{\infty}\left(M^{n} L^{2} \cdot(1+L \cdot)^{-1}\right)(\tau) \\
& =L(L-1)+\sum_{n=0}^{\infty} L^{2-n} \tau\left(1+L^{1-2 n} \tau\right) .
\end{aligned}
$$

It is easy to see that the series on the right hand side is absolutely convergent for any $\tau \in \mathbb{C}^{1}, \tau \neq-L^{2 n-1}, n=0,1, \ldots$, giving a meromorphic function. Let us notice that for $\tau \in]-L^{-1}, \infty[$

$$
t_{\infty}^{\prime}(\tau)=\sum_{n=0}^{\infty} L^{2-n}\left(1+L^{1-2 n} \tau\right)^{-2}>0,
$$


so that $t$ grows monotonically.

$$
\lim _{\tau \rightarrow-L^{-1}} t_{\infty}(\tau)=-\infty
$$

Now for $n_{0}$ being the largest integer with $L^{1-2 n_{0}} \tau \geqq 1$,

$$
\frac{1}{4} \sum_{n=0}^{n_{0}} L^{3 n} \tau^{-2} \leqq t_{\infty}^{\prime}(\tau) \leqq \sum_{n=0}^{n_{0}} L^{3 n} \tau^{-2}+\sum_{n=n_{0}+1}^{\infty} L^{2-n},
$$

or

$$
C_{1} \tau^{-1 / 2} \leqq t_{\infty}^{\prime}(\tau) \leqq C_{2} \tau^{-1 / 2},
$$

if $\tau$ is large. In particular

$$
\lim _{\tau>\infty} t_{\infty}(\tau)=\infty .
$$

We shall define $v_{\infty}^{\prime}$ by inverting $t_{\infty}$ on $]-L^{-1}, \infty\left[\right.$. Notice that $v_{\infty}^{\prime}\left(\varphi_{0}^{2}\right)=0, v_{\infty}^{\prime}$ grows from $-L^{-1}$ to $\infty$ when its argument runs from $-\infty$ to $\infty$. Thus $v_{\infty}$ has a single minimum at $\varphi_{0}^{2}$ and goes to $\infty$ when the arguments tend to $\pm \infty$. We shall normalize $v_{\infty}$ so that $v_{\infty}\left(\varphi_{0}^{2}\right)=0$. Of course $v_{\infty}$ is analytic around the real axis since $t_{\infty}$ was around $]-L^{-1}, \infty[$. In fact one can show that the analyticity region may be extended to include a small cone around the positive real axis. Since by (17)

we may write

$$
t_{\infty}^{\prime}(0)=\sum_{n=0}^{\infty} L^{2-n}=L^{3}(L-1)^{-1} \equiv \lambda_{\infty}^{-1}
$$

$$
v_{\infty}\left(\varphi_{0}^{2}+y\right) \equiv w_{\infty}(y)=\frac{1}{2} \lambda_{\infty} y^{2}+\tilde{\tilde{w}}_{\infty}(y),
$$

where $\tilde{\tilde{w}}_{\infty}(y)=O\left(y^{3}\right)$ for small $y$. Clearly the fixed point is non-Gaussian.

Notice that we could obtain other solutions of (10) and (11) by inverting $t$ on ]$-L^{2 n+1},-L^{2 n-1}\left[, n=0,1, \ldots\right.$. These however would give $v_{\infty}$ which is unbounded below violating stability and because of that uninteresting physically.

As noticed by $\mathrm{Ma}[14]$, if we write $t(\tau)-t_{\infty}(\tau)=\sum_{n=0}^{\infty} a_{n} \tau^{n}$, then, by virtue of (12),

$$
t_{1}(\tau)-t_{\infty}(\tau)=L\left[t\left(L^{-2} \tau\right)-t_{\infty}\left(L^{-2} \tau\right)\right]=\sum_{n=0}^{\infty} L^{1-2 n} a_{n} \tau^{n}
$$

Hence $a_{n}(v)$ are the scaling fields and the $L^{1-2 n}, n=0,1, \ldots$, are the eigenvalues of the linearized RG transformation around the non-Gaussian fixed point in the $N \rightarrow \infty$ limit. We clearly see the stability of this fixed point (only one eigenvalue is $>1$, the other corresponds to irrelevant directions). The aim of the subsequent sections is to prove that this picture remains correct for sufficiently large but finite $N$.

\section{Large $N$ Case. Invariant Neighborhood}

The corrections to the $N=\infty \mathrm{RG}$ transformation could be systematized into a perturbative $\frac{1}{N}$ expansion for the right hand side of (4.1). However, for the purpose 
of proving the existence of a fixed point for finite $N$, we shall need only crude bounds for the corrections.

Similarly, as in Sect. 2, the region of $\varphi^{2}$ near the minimum $\varphi_{0}^{2} \equiv L(L-1)^{-1}$ of the $N=\infty$ fixed point $v_{\infty}$ will be controlled more closely, the contributions from $\varphi^{2}$ away from $\varphi_{0}^{2}$ being small (of order $e^{-\delta N}$ ) and bounded with the use of the analyticity techniques. We shall normalize the single spin distributions $g\left(\varphi^{2}\right)$ so that $g\left(\varphi_{0}^{2}\right)=1$ and will introduce $\tilde{g}\left(\varphi_{0}^{2}\right)$ by

$$
g\left(\varphi_{0}^{2}+y\right) \equiv \tilde{g}\left(\varphi_{0}^{2}+y\right) \exp \left[-\frac{N}{2} a y\right]
$$

where $\tilde{g}^{\prime}\left(\varphi_{0}^{2}\right)=0$.

Let us state the assumptions for the factors $\tilde{g}$.

(a) $\tilde{g}\left(\varphi^{2}\right)$ is an analytic function for $|\operatorname{Im} \varphi|<\varrho$, positive for real $\varphi, \tilde{g}\left(\varphi_{0}^{2}\right)=1$, $\tilde{g}^{\prime}\left(\varphi_{0}^{2}\right)=0$.

(b) For $|y|<\varepsilon \equiv 2 \varphi_{0} \varrho$ (this is a different $\varepsilon$ as in Sect. 1-3)

$$
\tilde{g}\left(\varphi_{0}^{2}+y\right)=\exp \left[-\frac{N}{2} \tilde{w}(y)\right],
$$

where $\tilde{w}$ is a function analytic for $|y|<\varepsilon$. Writing

$$
\tilde{w}(y)=\frac{1}{2} \lambda y^{2}+\tilde{w}(y),
$$

where $\tilde{\tilde{w}}(0)=\tilde{w}^{\prime}(0)=\tilde{w}^{\prime \prime}(0)=0$, we have

$$
\begin{aligned}
& \left|\lambda-\lambda_{\infty}\right| \leqq \varepsilon^{3 / 2}, \\
& \sup _{|y|<\varepsilon}\left|\tilde{\tilde{w}}^{\prime \prime}\right| \leqq \varepsilon^{3 / 4} .
\end{aligned}
$$

(c) For $|\operatorname{Im} \varphi|<L^{-1 / 2} \varrho,|y| \geqq \varepsilon$, where $y \equiv \varphi^{2}-\varphi_{0}^{2}$,

$$
\left|\tilde{g}\left(\varphi^{2}\right)\right| \leqq \exp \left[-\frac{N}{11} \lambda_{\infty}(\operatorname{Re} y)^{2}+\frac{N}{2} \lambda_{\infty}(\operatorname{Im} y)^{2}\right] .
$$

Assumption (a) states the general properties of $\tilde{g}$. Notice that it means that $\tilde{g}(z)$ is analytic in the parabolic region $(\operatorname{Im} z)^{2}<4 \varrho^{2}\left(\operatorname{Re} z+\varrho^{2}\right)$. Assumption (b) implies that close to the minimum $\varphi_{0}^{2}$ of $v_{\infty}, \tilde{g}\left(\varphi^{2}\right)$ is a small perturbation of $\exp \left[-\frac{N}{2} v_{\infty}\left(\varphi^{2}\right)\right]$. Finally (c) is a convenient stability bound. The main result of the present section is

Proposition 3. If $\tilde{g}$ satisfies (a)-(c) and if $|a| \leqq 2 \varepsilon^{2}$, then $\tilde{g}_{1}$ also satisfies (a)-(c) provided that $L>L_{0}, \varrho<\varrho_{0}(L)$, and $N \geqq N_{0}(L, \varrho)$.

Proof. Introducing the notation

$$
\begin{aligned}
G\left(s, u, \varphi^{2}\right) \equiv & g\left(L^{-1} \varphi^{2}+2 L^{-1 / 2} \varphi s+s^{2}+u\right)^{\frac{1}{2} L^{3}} g\left(L^{-1} \varphi^{2}-2 L^{-1 / 2} \varphi s+s^{2}+u\right)^{\frac{1}{2} L^{3}} \\
& \cdot \exp \left[-\frac{N}{2}\left(s^{2}+u-\log u\right)-\frac{3}{2} \log u\right],
\end{aligned}
$$


we may rewrite (1) as

$$
g_{1}\left(\varphi^{2}\right)=\int_{-\infty}^{\infty} d s \int_{0}^{\infty} d u G\left(s, u, \varphi^{2}\right) / \int_{-\infty}^{\infty} d s \int_{0}^{\infty} d u G\left(s, u, \varphi_{0}^{2}\right)
$$

Notice that by virtue of (a)-(c) the integrals in (8) converge absolutely uniformly in $\varphi$ such that $|\operatorname{Im} \varphi|<\varrho$ [since for such $\varphi$ the arguments of $g$ in (7) have an imaginary part bounded by $L^{-1 / 2} \varrho$ ]. Hence (a) for $\tilde{g}_{1}$ follows immediately.

In order to study (b), take $\varphi^{2} \equiv \varphi_{0}^{2}+y$.

$N=\infty$ Recursion. Let us define

$$
w(y) \equiv a y+\tilde{w}(y),
$$

see (2). The large $N$ limit approximation $w_{1 \infty}(y)$ to $w_{1}(y)$ is given by (4.10) and (4.11) which, after translation by $\varphi_{0}^{2}$ to rewrite them in terms of $w$ 's, read

$$
\begin{gathered}
w_{1 \infty}^{\prime}(y)=L^{2} w^{\prime}\left(L^{-1} y+u_{0}-1\right), \\
L^{3} w^{\prime}\left(L^{-1} y+u_{0}-1\right)+1-\frac{1}{u_{0}}=0 .
\end{gathered}
$$

In the first, rough attempt, we take $w^{\prime}(y)=a+\lambda y$ and compute $u_{0}$ from (11) approximating $\frac{1}{u_{0}} \approx 1-\left(u_{0}-1\right)$. This gives

$$
u_{0}-1=-L^{3}\left(1+L^{3} \lambda\right)^{-1} a-L^{2} \lambda\left(1+L^{3} \lambda\right)^{-1} y+\tilde{u},
$$

where $\tilde{u}$ is the correction due to the approximate character of the calculation. We shall search for the solution of (11) for small $y$ in the form (12). This is a standard problem.

Lemma 1. For $|y|<\frac{3}{4} L^{2} \varepsilon$, there exists a unique analytic function $\tilde{u}(y)$ satisfying (11) and (12) and such that $|\tilde{u}(y)| \leqq \frac{4}{3} L^{2} \varepsilon^{7 / 4}$. Moreover $\left|\tilde{u}^{\prime}(y)\right| \leqq \frac{4}{3} \varepsilon^{3 / 4},|\tilde{u}(0)| \leqq O\left(\varepsilon^{15 / 4}\right)$, and $\left|\tilde{u}^{\prime}(0)\right| \leqq O\left(\varepsilon^{7 / 4}\right)$.

Proof of Lemma 1. Inserting (12) to (11) and using (3) and (9) we obtain

$$
\begin{aligned}
\tilde{u}= & -\left(1+L^{3} \lambda\right)^{-1}\left[L^{3} \tilde{w}^{\prime}\left(-L^{3}\left(1+L^{3} \lambda\right)^{-1} a+L^{-1}\left(1+L^{3} \lambda\right)^{-1} y+\tilde{u}\right)\right. \\
& +1+L^{3}\left(1+L^{3} \lambda\right)^{-1} a+L^{2} \lambda\left(1+L^{3} \lambda\right)^{-1} y-\tilde{u}-\left(1-L^{3}\left(1+L^{3}\right) \lambda\right)^{-1} a \\
& \left.\left.-L^{2} \lambda\left(1+L^{3} \lambda\right)^{-1} y+\tilde{u}\right)^{-1}\right] \equiv F(y, \tilde{u}) .
\end{aligned}
$$

Notice that by virtue of (4),

$$
L^{-1}\left(1+L^{3} \lambda\right)^{-1} y=\left(L^{-2}+O\left(\varepsilon^{3 / 2}\right)\right) y .
$$

Hence it is easy to see that for $|y|<\frac{3}{4} L^{2} \varepsilon, F(y, \cdot)$ maps, say, $K\left(0, \varepsilon^{3 / 2}\right)$ in $\tilde{u}$ into $K\left(0, \frac{4}{3} L^{2} \varepsilon^{7 / 4}\right)$ ( $\varepsilon$ is small). Its derivative over $\tilde{u}$ on the same ball is bounded by $O\left(\varepsilon^{3 / 4}\right)$. Thus there exists a unique solution of $(13), \tilde{u}(y)$, in $\left.\overline{K\left(0, \frac{3}{4} L^{2} \varepsilon^{7 / 4}\right.}\right)$ which may be constructed by successive approximations. For $y=0, F(0, \cdot) \operatorname{maps} K\left(0, \varepsilon^{2}\right)$ into $K\left(0,0\left(\varepsilon^{15 / 4}\right)\right.$ ) [to see this notice that by virtue of the maximum principle, $\left|\tilde{w}^{\prime}(z)\right|$ $\leqq|z / \varepsilon|^{2} \varepsilon^{7 / 4}$ for $|z|<\varepsilon$ since $\left.\tilde{\tilde{w}}^{\prime}(0)=\tilde{\tilde{w}}^{\prime \prime}(0)=0\right]$. This gives $|\tilde{u}(0)| \leqq O\left(\varepsilon^{15 / 4}\right)$. Moreover 


$$
\left|\frac{\partial F}{\partial \tilde{u}}(y, \tilde{u}(y))\right| \leqq \frac{7}{6} L^{2} \varepsilon^{3 / 4}, \quad\left|\frac{\partial F}{\partial y}(y, \tilde{u}(y))\right| \leqq \frac{7}{6} \varepsilon^{3 / 4}
$$

and

$$
\left|\frac{\partial F}{\partial \tilde{u}}(0, \tilde{u}(0))\right| \leqq O\left(\varepsilon^{7 / 4}\right), \quad\left|\frac{\partial F}{\partial y}(0, \tilde{u}(0))\right| \leqq O\left(\varepsilon^{7 / 4}\right) .
$$

Hence

$$
\left|\tilde{u}^{\prime}(y)\right|=\left|\frac{\partial F}{\partial y}(y, \tilde{u}(y)) /\left(1-\frac{\partial F}{\partial \tilde{u}}(y, \tilde{u}(y))\right)\right| \leqq \frac{4}{3} \varepsilon^{3 / 4}
$$

and

$$
\left|\tilde{u}^{\prime}(0)\right| \leqq O\left(\varepsilon^{7 / 4}\right),
$$

which ends Proof of Lemma 1.

Given Lemma 1, we may extract the properties of the function $w_{1 \infty}^{\prime}(y)$ analytic for $|y|<\frac{3}{4} L^{2} \varepsilon$ as given by (10) and (12):

$$
w_{1 \infty}^{\prime}(y)=L^{2} w^{\prime}\left(-L^{3}\left(1+L^{3} \lambda\right)^{-1} a+L^{-1}\left(1+L^{3} \lambda\right)^{-1} y+\tilde{u}(y)\right) .
$$

We write

$$
w_{1 \infty}^{\prime}(y)=a_{1 \infty}+\lambda_{1 \infty} y+\tilde{\tilde{w}}_{1 \infty}^{\prime}(y)
$$

where $\tilde{\tilde{w}}_{1 \infty}^{\prime}(0)=\tilde{\tilde{w}}_{1 \infty}^{\prime \prime}(0)=0$, and obtain

$$
\begin{aligned}
a_{1 \infty}= & L^{2} w^{\prime}\left(-L^{3}\left(1+L^{3} \lambda\right)^{-1} a+\tilde{u}(0)\right)=L^{2} a-L^{5} \lambda\left(1+L^{3} \lambda\right)^{-1} a \\
& +L^{2} \lambda \tilde{u}(0)+L^{2} \tilde{\tilde{w}}^{\prime}\left(-L^{3}\left(1+L^{3} \lambda\right)^{-1} a+\tilde{u}(0)\right)=L a+O\left(\varepsilon^{7 / 2}\right), \\
\lambda_{1 \infty}= & L \lambda\left(1+L^{3} \lambda\right)^{-1}+L^{2} \lambda \tilde{u}^{\prime}(0)+L^{2} \tilde{\tilde{w}}^{\prime \prime}\left(-L^{3}\left(1+L^{3} \lambda\right)^{-1} a+\tilde{u}(0)\right) \\
& \cdot\left(L^{-1}\left(1+L^{3} \lambda\right)^{-1}+\tilde{u}^{\prime}(0)\right)=L \lambda\left(1+L^{3} \lambda\right)^{-1}+O\left(\varepsilon^{7 / 4}\right) \\
= & \lambda_{\infty}+L^{-1}\left(\lambda-\lambda_{\infty}\right)+O\left(\varepsilon^{7 / 4}\right) .
\end{aligned}
$$

Equations (21) and (22) describe the behavior of the relevant and the least irrelevant perturbation near the $N=\infty$ fixed point in agreement with the analysis of the end of Sect. 4 of the linearized RG. Finally, for $|y|<\frac{3}{4} L^{2} \varepsilon$,

$$
\begin{aligned}
\left|\tilde{\tilde{w}}_{1 \infty}^{\prime \prime}(y)\right|= & \mid L^{2} \lambda \tilde{u}^{\prime}(y)+L^{2} \tilde{\tilde{w}}^{\prime \prime}\left(-L^{3}\left(1+L^{3} \lambda\right)^{-1} a+L^{-1}\left(1+L^{3} \lambda\right)^{-1} y+\tilde{u}(y)\right) \\
& \cdot\left(L^{-1}\left(1+L^{3} \lambda\right)^{-1}+\tilde{u}^{\prime}(y)\right)-L^{2} \lambda \tilde{u}^{\prime}(0)-L^{2} \tilde{\tilde{w}}^{\prime \prime}\left(-L^{3}\left(1+L^{3} \lambda\right)^{-1} a+\tilde{u}(0)\right) \\
& \cdot\left(L^{-1}\left(1+L^{3} \lambda\right)^{-1}+\tilde{u}^{\prime}(0)\right) \mid \leqq \frac{5}{2} \varepsilon^{3 / 4}
\end{aligned}
$$

Using $\left|\tilde{\tilde{w}}_{1 \infty}^{\prime \prime}(z)\right| \leqq\left|z /\left(\frac{3}{4} L^{2} \varepsilon\right)\right| \sup \left|\tilde{\tilde{w}}_{1 \infty}^{\prime \prime}\right|$, due to the vanishing of $\tilde{\tilde{w}}_{1 \infty}^{\prime \prime}$ at zero and the maximum principle, we obtain from (23)

$$
\left|\tilde{\tilde{w}}_{1 \infty}^{\prime \prime}(y)\right| \leqq\left(\frac{3}{4} L^{2}\right)^{-1} \frac{5}{2} \varepsilon^{3 / 4} \leqq \frac{3}{4} \varepsilon^{3 / 4} \text { for }|y|<\varepsilon,
$$

$\left(L>L_{0}\right)$. Equations (22) and (24) show that $\tilde{w}_{1 \infty}\left(=\lambda_{1 \infty} y+\tilde{\tilde{w}}_{1 \infty}(y)\right)$ satisfies (b) with some space to accomodate the finite $N$ corrections. 
Small Field Integral. In this subsection we shall consider $\varphi^{2} \equiv \varphi_{0}^{2}+y$ and $|y|<\varepsilon$ or $|\operatorname{Im} \varphi|<L^{-1 / 2} \varrho, \quad|\operatorname{Re} y|<L^{1 / 2} \varepsilon$. Notice that if $|y|<\varepsilon \equiv 2 \varphi_{0} \varrho$, then $\varphi^{2}=\varphi_{0}^{2}+r \cos \theta+i r \sin \theta$ with $0 \leqq r<2 \varphi_{0} \varrho$ and

$$
\begin{aligned}
r^{2} \sin ^{2} \theta & =\left[\operatorname{Im}\left(2 \varrho^{2}+r \cos \theta+i r \sin \theta\right)\right]^{2} \leqq\left|2 \varrho^{2}+r \cos \theta+i r \sin \theta\right|^{2} \\
& =4 \varrho^{4}+4 \varrho^{2} r \cos \theta+r^{2}<4 \varrho^{2}\left(\varphi_{0}^{2}+r \cos \theta+\varrho^{2}\right) .
\end{aligned}
$$

But this inequality implies that $|\operatorname{Im} \varphi|<\varrho$, so that (8) is well defined for $\varphi^{2}=\varphi_{0}^{2}+y$ and $|y|<\varepsilon$.

We shall divide the integral of (8) into two contributions. To this end define

$$
\mathcal{O}=\left\{(s, u) \in \mathbb{C}^{2}:|s|<\frac{1}{2} \varepsilon, \quad\left|u-1+\left(L^{-1}-L^{-2}\right) \operatorname{Re} y\right|<\frac{1}{2} \varepsilon\right\},
$$

and write

$$
\int_{-\infty}^{\infty} d s \int_{0}^{\infty} d u G\left(s, u, \varphi^{2}\right)=\int_{\operatorname{Re} \mathcal{O}} d s d u G\left(s, u, \varphi^{2}\right)+\int_{\left(\mathbb{R}^{1} \times \mathbb{R}_{+}^{1}\right) \backslash \mathcal{O}} d s d u G\left(s, u, \varphi^{2}\right) .
$$

The first integral is the main contribution. Notice that, by virtue of (7), (b) and (9), for $(s, u) \in \mathcal{O}$

$$
G\left(s, u, \varphi_{0}^{2}+y\right)=\exp \left[-\frac{N}{2} V(s, u, y)\right]
$$

where

$$
\begin{aligned}
V(s, u, y)= & \frac{1}{2} L^{3} w\left(L^{-1} y+2 L^{-1 / 2} \varphi s+s^{2}+u-1\right)+\frac{1}{2} L^{3} w\left(L^{-1} y\right. \\
& \left.-2 L^{-1 / 2} \varphi s+s^{2}+u-1\right)+s^{2}+u-\left(1-\frac{3}{N}\right) \log u
\end{aligned}
$$

Indeed

$$
\begin{gathered}
\left|L^{-1} y \pm 2 L^{-1 / 2} \varphi s+s^{2}+u-1\right| \leqq L^{-2}|\operatorname{Re} y|+L^{-1}|\operatorname{Im} y|+2 L^{-1 / 2} \varphi_{0}|s| \\
+\frac{1}{2} \varepsilon+O\left(\varepsilon^{2}\right)<\varepsilon\left(L>L_{0}, \varepsilon<\varepsilon_{0}(L)\right)
\end{gathered}
$$

so that we are in the domain where (2) applies. Notice that $\left(0, u_{0}(y)\right)$, as given by (12) and Lemma 1, is deep inside $\mathcal{O}$ for $L$ big and that

$$
w_{1 \infty}(y)=V\left(0, u_{0}(y), y\right)-V\left(0, u_{0}(0), 0\right)-\frac{3}{N}\left(\log u_{0}(y)-\log u_{0}(0)\right) .
$$

To exhibit the $N=\infty$ contribution (29) to $\int_{\operatorname{Re} \mathscr{O}} d s d u G\left(s, u, \varphi^{2}\right)$, we shall change the contour of integration over $u$ to the one given by $C A B$ on Fig. 1 . This way we obtain

$$
\begin{aligned}
\int_{\operatorname{Re} \mathcal{O}} d s d u G\left(s, u, \varphi^{2}\right)= & \exp \left[-\frac{N}{2} V\left(0, u_{0}(y), y\right)\right] \\
& \cdot \int_{-\alpha}^{\alpha} d s \int_{C A B} d u \exp \left[-\frac{N}{2}\left(V(s, u, y)-V\left(0, u_{0}(y), y\right)\right)\right],
\end{aligned}
$$




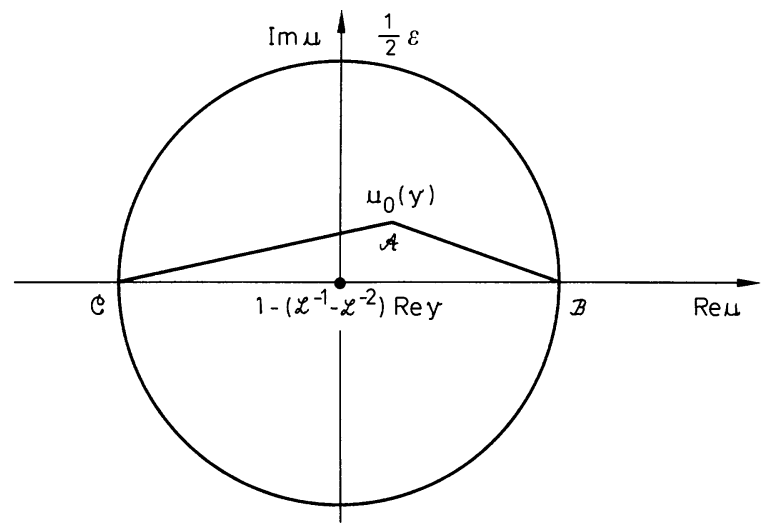

where $\alpha \equiv \frac{1}{2} \varepsilon$. The integral on the right hand side of (30) is a small perturbation of a Gaussian integral of the type $\int \exp \left[-\frac{N}{2}(\sigma \mid \mathscr{A} \sigma)\right] d^{2} \sigma$ with a positive matrix $\mathscr{A}$. It is estimated in

\section{Lemma 2.}

$$
\int_{-\alpha}^{\alpha} d s \int_{C A B} d u \exp \left[-\frac{N}{2}\left(V(s, u, y)-V\left(0, u_{0}(y), y\right)\right)\right]=\pi\left(\frac{8}{3 L}\right)^{1 / 2} N^{-1}+0\left(\varepsilon^{3 / 4} N^{-1}\right) \text {. }
$$

Proof of Lemma 2. First let us notice that on $\mathcal{O}$

$$
\left(\begin{array}{ll}
\frac{\partial^{2} V}{\partial s^{2}}, & \frac{\partial^{2} V}{\partial s \partial u} \\
\frac{\partial^{2} V}{\partial u \partial s}, & \frac{\partial^{2} V}{\partial u^{2}}
\end{array}\right) \quad(s, u, y)=\left(\begin{array}{ll}
6 & 0 \\
0 & L
\end{array}\right)+O\left(\varepsilon^{3 / 4}\right)
$$

$\left(N>N_{0}(\varepsilon)\right)$. For the interval $A B$, let us parametrize

$$
\begin{gathered}
s=\alpha \sigma_{1}, \\
u=u_{0}(y)+\sigma_{2}(B-A),
\end{gathered}
$$

with $\left|\sigma_{1}\right|<1,0<\sigma_{2}<1$. This allows us to write

$$
\begin{aligned}
& \int_{-\alpha}^{\alpha} d s \int_{A B} d u \exp \left[-\frac{N}{2}\left(V(s, u, y)-V\left(s, u_{0}(y), y\right)\right)\right] \\
& =\alpha(B-A) \int_{-1}^{1} d \sigma_{1} \int_{0}^{1} d \sigma_{2} \exp \left[-\frac{N}{2}(\sigma \mid \mathscr{A} \sigma)-N U(\sigma)+O(\varepsilon)\right],
\end{aligned}
$$

where the matrix

$$
\begin{aligned}
\mathscr{A} & =\frac{1}{2}\left(\begin{array}{cc}
\alpha^{2} \frac{\partial^{2} V}{\partial s^{2}}, & \alpha(B-A) \frac{\partial^{2} V}{\partial s \partial u} \\
\alpha(B-A) \frac{\partial^{2} V}{\partial s \partial u},(B-A)^{2} \frac{\partial^{2} V}{\partial u^{2}}
\end{array}\right)\left(0, u_{0}(y), y\right) \\
& =\left(\begin{array}{cc}
3 \alpha^{2} & 0 \\
0 & \frac{1}{2}(B-A)^{2} L
\end{array}\right)+O\left(\varepsilon^{11 / 4}\right)
\end{aligned}
$$


and the higher order contribution

$$
|U(\sigma)| \leqq O\left(\varepsilon^{11 / 4}\right)|\sigma|^{3},
$$

see (32) and (33). We use the fact that

$$
\frac{\partial V}{\partial s}\left(0, u_{0}(y), y\right)=\frac{\partial V}{\partial u}\left(0, u_{0}(y), y\right)-\frac{3}{N} u_{0}(y)^{-1}=0,
$$

since $u_{0}(y)$ was defined by means of these relations. We also have

$$
\operatorname{Re}\left[\frac{1}{2}(\sigma \mid \mathscr{A} \sigma)+\mathrm{U}(\sigma)\right] \geqq \frac{1}{4} \operatorname{Re}(\sigma \mid \mathscr{A} \sigma),
$$

since $(B-A)^{2}$ has a small argument $(A$ is deep inside $\mathcal{O}$ for $L$ big). Defining: $U\left(\sigma_{1},-\sigma_{2}\right) \equiv U\left(\sigma_{1}, \sigma_{2}\right)$, we may write

$$
\begin{aligned}
\alpha(B-A) & \int_{-1}^{1} d \sigma_{1} \int_{0}^{1} d \sigma_{2} \exp \left[-\frac{N}{2}(\sigma \mid \mathscr{A} \sigma)-N U(\sigma)\right] \\
= & \frac{1}{2} \alpha(B-A) \int_{\left|\sigma_{1}\right|,\left|\sigma_{2}\right|<1} d^{2} \sigma \exp \left[-\frac{N}{2}(\sigma \mid \mathscr{A} \sigma)\right] \\
& +\frac{1}{2} \alpha(B-A) \int_{|\sigma|<N^{-2 / 5}} d^{2} \sigma \exp \left[-\frac{N}{2}(\sigma \mid \mathscr{A} \sigma)\right](\exp [-N U(\sigma)]-1) \\
& +\frac{1}{2} \alpha(B-A) \int_{\substack{|\sigma| \geq N-2 / 5 \\
|\sigma 1||,| \sigma_{2} \mid<1}} d^{2} \sigma \exp \left[-\frac{N}{2}(\sigma \mid \mathscr{A} \sigma)\right](\exp [-N U(\sigma)]-1) .(38)
\end{aligned}
$$

The first term on the right hand side of (38) is

$$
\pi \alpha(B-A)(\operatorname{det} \mathscr{A})^{-1 / 2} N^{-1}+O\left(e^{-\delta N}\right)
$$

for some $\delta>0$. The second one is bounded by $O\left(\varepsilon^{11 / 4} N^{-6 / 5}\right)$ by virtue of (36), and finally the third one by $O\left(e^{-\delta N^{1 / 5}}\right)$. Altogether it produces the estimate

$$
(38)=\left(\frac{2}{3 L}\right)^{1 / 2} \pi N^{-1}+O\left(\varepsilon^{3 / 4} N^{-1}\right) \text {. }
$$

Substituting this and the analogous estimate for the contribution of the $C A$ interval to (34), we obtain (31).

Equation (30) and Lemma 2 imply that

$$
\int_{\operatorname{Re} \mathcal{O}} d s d u G\left(s, u, \varphi^{2}\right)=\exp \left[-\frac{N}{2} V\left(0, u_{0}(y), y\right)\right]\left(\pi\left(\frac{8}{3 L}\right)^{1 / 2}+O\left(\varepsilon^{3 / 4}\right)\right) N^{-1} .
$$

Large Field Integral. In this subsection we shall estimate the second term on the right hand side of (26) for the region of the values of $\varphi^{2}$ (of $y$ ) as in the previous one (see its beginning). The aim is to show that this term is down by a factor $e^{-\delta N}$ as compared with the first one. To exhibit this we shall prove certain inequalities for $\left|G\left(s, u, \varphi^{2}\right)\right|$ with real $s, u$ and $|\operatorname{Im} \varphi|<\varrho$ which will also be used in the later arguments.

First notice that by virtue of (b), (c) and the assumption on $a$,

$$
\left|g\left(\varphi^{2}\right)\right| \leqq \exp \left[-\frac{N}{12} \lambda_{\infty}(\operatorname{Re} y)^{2}+\frac{N}{2} \lambda_{\infty}(\operatorname{Im} y)^{2}+N O\left(\varepsilon^{11 / 4}\right)\right]
$$


for $|\operatorname{Im} \varphi|<L^{-1 / 2} \varrho$. But the arguments of $g$ in (7) fulfill this requirement for real $s, u$ and $|\operatorname{Im} \varphi|<\varrho$. Hence

$$
\begin{aligned}
\left|G\left(s, u, \varphi^{2}\right)\right| \leqq & \exp \left[-\frac{N}{12} L \lambda_{\infty}(\operatorname{Re} y)^{2}+\frac{N}{2} L \lambda_{\infty}(\operatorname{Im} y)^{2}+N O\left(\varepsilon^{11 / 4}\right)\right] \\
& \cdot \exp \left[-\frac{N}{6} L^{2} \lambda_{\infty} \operatorname{Re} y\left(s^{2}+u-1\right)-\frac{N}{12} L^{3} \lambda_{\infty}\left(s^{2}+u-1\right)^{2}\right. \\
& \left.-\frac{N}{3} L^{2} \lambda_{\infty}(\operatorname{Re} \varphi)^{2} s^{2}+2 N L^{2} \lambda_{\infty}(\operatorname{Im} \varphi)^{2} s^{2}\right] \\
& \cdot \exp \left[-\frac{N}{2}\left(s^{2}+u-\log u\right)-\frac{3}{2} \log u+N O\left(\varepsilon^{11 / 4}\right)\right]
\end{aligned}
$$

Now, for $0<u<\frac{3}{2}$,

$$
\frac{N}{2}(u-\log u)+\frac{3}{2} \log u \geqq \frac{N}{2}+\frac{N}{6}(u-1)^{2}-O(1) .
$$

Moreover,

$$
\begin{aligned}
\frac{N}{6} L^{2} \lambda_{\infty} \operatorname{Re} y\left(s^{2}+u-1\right)+\frac{N}{12} L^{3} \lambda_{\infty}\left(s^{2}+u-1\right)^{2}+\frac{N}{6}(u-1)^{2} \\
=\frac{N}{6}\left(u-1+L^{2} \lambda_{\infty}\left(2+L^{3} \lambda_{\infty}\right)^{-1} \operatorname{Re} y\right)^{2}+\frac{N}{12} L^{3} \lambda_{\infty}\left(s^{2}+u-1+L^{2} \lambda_{\infty}\right. \\
\left.\quad \cdot\left(2+L^{3} \lambda_{\infty}\right)^{-1} \operatorname{Re} y\right)^{2}-\frac{N}{12} L^{4} \lambda_{\infty}^{2}\left(2+L^{3} \lambda_{\infty}\right)^{-1}(\operatorname{Re} y)^{2} \\
\quad+\frac{N}{3} L^{2} \lambda_{\infty}\left(2+L^{3} \lambda_{\infty}\right)^{-1} \operatorname{Re} y s^{2} \geqq \frac{N}{6}\left(u-1+L^{2} \lambda_{\infty}\left(2+L^{3} \lambda_{\infty}\right)^{-1} \operatorname{Re} y\right)^{2} \\
\quad-\frac{N}{12} L^{4} \lambda_{\infty}^{2}\left(2+L^{3} \lambda_{\infty}\right)^{-1}(\operatorname{Re} y)^{2}-\frac{N}{12} s^{2},
\end{aligned}
$$

as a simple algebra shows. Hence, for $0<u<\frac{3}{2}$, real $s$ and $|\operatorname{Im} \varphi|<\varrho$,

$$
\begin{aligned}
\left|G\left(s, u, \varphi^{2}\right)\right| \leqq & \exp \left[-\frac{N}{6} L\left(2+L^{3} \lambda_{\infty}\right)^{-1} \lambda_{\infty}(\operatorname{Re} y)^{2}+\frac{N}{2} L \lambda_{\infty}(\operatorname{Im} y)^{2}\right. \\
& \left.-\frac{N}{6}\left(u-1+L^{2} \lambda_{\infty}\left(2+L^{3} \lambda_{\infty}\right)^{-1} \operatorname{Re} y\right)^{2}-\frac{N}{3} s^{2}-\frac{N}{2}+N O\left(\varepsilon^{11 / 4}\right)\right]
\end{aligned}
$$

For $u \geqq \frac{3}{2}$ the second and the third exponent on the right hand side of (43) decrease with $u$ as is easily seen. Hence for $u \geqq \frac{3}{2}$, real $s,|\operatorname{Im} \varphi|<\varrho$

$\left|G\left(s, u, \varphi^{2}\right)\right| \leqq$ right hand side of $\left.(46)\right|_{u=\frac{3}{2}}$

$$
\cdot \exp \left[-\frac{N}{2}(u-\log u)-\frac{3}{2} \log u+\frac{N}{2}\left(\frac{3}{2}-\log \frac{3}{2}\right)+\frac{3}{2} \log \frac{3}{2}\right] .
$$


Inequalities (46), (47), and (25) imply that for $\varphi^{2}=\varphi_{0}^{2}+y$ with $|y|<\varepsilon$ or $|\operatorname{Im} \varphi|<L^{-1 / 2} \varrho,|\operatorname{Re} y|<L^{1 / 2} \varepsilon$,

$\int_{\left(\mathbb{R}^{1} \times \mathbb{R}_{+}^{1}\right) \backslash 0} d s d u\left|G\left(s, u, \varphi^{2}\right)\right| \leqq \exp \left[\frac{N}{2} L \lambda_{\infty}(\operatorname{Im} y)^{2}-\frac{N}{30} \varepsilon^{2}-\frac{N}{2}+N O\left(\varepsilon^{11 / 4}\right)\right]$.

In order to compare (48) with (41) notice that by (29), (20)-(23), (28), (14) and Lemma 1 ,

$$
\begin{aligned}
V\left(0, u_{0}(y), y\right)= & a_{1 \infty} y+\frac{1}{2} \lambda_{1 \infty} y^{2}+\tilde{\tilde{w}}_{1 \infty}(y)+L^{3} \tilde{\tilde{w}}\left(u_{0}(0)-1\right) \\
& +u_{0}(0)-\log u_{0}(0)+\frac{3}{N} \log u_{0}(y)=1+\frac{1}{2} \lambda_{\infty} y^{2}+O\left(\varepsilon^{11 / 4}\right) .
\end{aligned}
$$

Hence we easily see that

$$
\int_{\left(\mathbb{R}^{1} \times \mathbb{R}_{+}^{1}\right) \backslash \mathcal{O}}\left|G\left(s, u, \varphi^{2}\right)\right| \leqq\left|\int_{\operatorname{Re} \mathcal{O}} G\left(s, u, \varphi^{2}\right)\right| e^{-\delta N}
$$

for $L, \varepsilon^{-1}, N$ big and some $\delta>0$. Finally

$$
\begin{aligned}
g_{1}\left(\varphi^{2}\right) & =\int_{\mathbb{R}^{1} \times \mathbb{R}_{+}^{1}} d s d u G\left(s, u, \varphi^{2}\right) / \int_{\mathbb{R}^{1} \times \mathbb{R}_{+}^{1}} d s d u G\left(s, u, \varphi_{0}^{2}\right) \\
& =\exp \left[-\frac{N}{2}\left(V\left(0, u_{0}(y), y\right)-V\left(0, u_{0}(0), 0\right)\right)\right] \exp \left[O\left(\varepsilon^{3 / 4}\right)+O\left(e^{-\delta N}\right)\right] \\
& =\exp \left[-\frac{N}{2} w_{1 \infty}(y)+O\left(\varepsilon^{3 / 4}\right)\right]
\end{aligned}
$$

$\left(N>N_{0}(\varepsilon)\right)$. Thus we obtain

$$
w_{1}(y)=w_{1 \infty}(y)+O\left(\varepsilon^{3 / 4} N^{-1}\right) .
$$

This together with (20)-(22) and (24) proves (b) for $\tilde{g}_{1}$ and shows that

$$
a_{1}=L a+O\left(\varepsilon^{7 / 2}\right) \text {. }
$$

At the same time (6) of (c) follows for $\varphi^{2}=\varphi_{0}^{2}+y$ with $|\operatorname{Im} \varphi|<L^{-1 / 2} \varrho,|y| \geqq \varepsilon$, and $|\operatorname{Re} y|<L^{1 / 2} \varepsilon$.

We are left with showing (6) for $\varphi^{2}$ with $|\operatorname{Im} \varphi|<L^{-1 / 2} \varrho$ and $|\operatorname{Re} y| \geqq L^{1 / 2} \varepsilon$. This bound iterates because the factor at $-\frac{N}{2}(\operatorname{Re} y)^{2}$ in the exponent has a value smaller than at the fixed point and thus grows under the RG recursion. Indeed, (46) and (47) imply that

$$
\begin{aligned}
& \left|\int_{\mathbb{R}^{1} \times \mathbb{R}_{+}^{1}} d s d u G\left(s, u, \varphi^{2}\right)\right| \\
& \quad \leqq \exp \left[-\frac{N}{6} L\left(2+L^{3} \lambda_{\infty}\right)^{-1} \lambda_{\infty}(\operatorname{Re} y)^{2}+\frac{N}{2} L \lambda_{\infty}(\operatorname{Im} y)^{2}-\frac{N}{2}+N O\left(\varepsilon^{1 / 4}\right)\right] .
\end{aligned}
$$

Moreover by (41), (49), and (50)

$$
\left|\int_{\mathbb{R}^{1} \times \mathbb{R}_{+}^{1}} d s d u G\left(s, u, \varphi_{0}^{2}\right)\right| \geqq \exp \left[-\frac{N}{2}-N O\left(\varepsilon^{11 / 4}\right)\right] .
$$


Hence

$$
\left|g_{1}\left(\varphi^{2}\right)\right| \leqq \exp \left[-\frac{N}{6}\left(1+L^{-1}\right)^{-1} \lambda_{\infty}(\operatorname{Re} y)^{2}+\frac{N}{2} L \lambda_{\infty}(\operatorname{Im} y)^{2}+N O\left(\varepsilon^{1 / 4}\right)\right],
$$

which establishes the missing part of (c) for $\tilde{g}_{1}\left(\varphi^{2}\right)$, since for $|\operatorname{Im} \varphi|<L^{-1 / 2} \varrho$, $|\operatorname{Re} y| \geqq L^{1 / 2} \varepsilon$,

$$
|\operatorname{Im} y|<\frac{4}{3} L^{-1}|\operatorname{Re} y|
$$

[we also use the fact that $\left|a_{1}\right| \leqq O\left(\varepsilon^{2}\right)$ ]. This ends the Proof of Proposition 3 .

\section{Large $N$ Case. Contractive Properties of the RG Recursion}

From (5.53) we see that in general there is no contraction of the coefficient $a$ under the $\mathrm{RG}$ transformation (4.1) $\left[a\left(\varphi^{2}-\varphi_{0}^{2}\right)\right.$ is a relevant perturbation]. However, similarly as for the small $\varepsilon$ case in Sect. 3, we shall show that for each $\tilde{g}$ satisfying (a)-(c) there exists a critical value of $a$ for which the assumptions of Proposition 3 are stable under (4.1). Moreover for this value of $a$ the "distance" between the subsequent iterates of (4.1) shrinks, yielding a convergence to a non-Gaussian fixed point.

The strategy of search for the critical value is the same as in Sect. 3. We shall restrict the admissible values of $a$ gradually from one iteration of (4.1) to the other so that finally the critical $a$ (corresponding to the critical value of the temperature) is chosen.

Let us start with $g_{0}\left(\varphi^{2}\right)=\tilde{g}_{0}\left(\varphi^{2}\right) \exp \left[-\frac{N}{2} a_{0} y\right]$. Suppose that $\tilde{g}_{0}$ fulfills (a)-(c) of Sect. 5 and that $a_{0} \in\left[-\varepsilon^{2}, \varepsilon^{2}\right]$. From (5.53) it follows that one can choose a closed interval $I_{0} \subset\left[-\varepsilon^{2}, \varepsilon^{2}\right]$ such that $\Delta a_{0} \equiv a_{1}-a_{0}$ sweeps, say, $\left[-\varepsilon^{3}, \varepsilon^{3}\right]$, when $a_{0}$ runs through $I_{0}$. Let $g_{n}$ be the $n^{\text {th }}$ iterate of (4.1) applied to $g_{0}$. The following are the properties of $g_{n}$ and $g_{n+1}$ to be shown inductively. They are obviously fulfilled by $g_{0}$ and $g_{1}$. We use the notation $\Delta f_{n} \equiv f_{n+1}-f_{n}$.

$\left(\mathrm{A}_{n}\right)$. For $0 \leqq k \leqq n$ one can choose a closed interval $I_{k} \subset\left[-\varepsilon^{2}, \varepsilon^{2}\right], I_{k+1} \subset I_{k}$, such that $\Delta a_{k}$ sweeps $\left.L^{-k / 2}\right]-\varepsilon^{3}, \varepsilon^{3}\left[\right.$ when $a_{0}$ runs through $I_{k}$.

$\left(\mathrm{B}_{n}\right)$. For $a \in I_{n}$ and $|y|<\varepsilon$

$$
\left|\Delta \lambda_{n}\right| \leqq 2 L^{-n / 2} \varepsilon^{3 / 2}
$$

and

$$
\left|\Delta \tilde{\tilde{w}}_{n}^{\prime \prime}\right| \leqq 2 L^{-n / 2} \varepsilon^{3 / 4}
$$

$\left(\mathrm{C}_{n}\right)$. For $|\operatorname{Im} \varphi|<L^{-1 / 2} \varrho,|y| \geqq \varepsilon$,

$$
\left|\Delta \tilde{g}_{n}\left(\varphi_{0}^{2}+y\right)\right| \leqq N L^{-n / 2} \exp \left[-\frac{N}{11} \lambda_{\infty}(\operatorname{Re} y)^{2}+\frac{N}{2} \lambda_{\infty}(\operatorname{Im} y)^{2}\right] .
$$

We shall prove the following:

Proposition 4. Let $L>L_{0}, \varrho<\varrho_{0}(L), \quad N>N_{0}(L, \varrho)$. Then $\left(\mathrm{A}_{n}\right)-\left(\mathrm{C}_{n}\right)$ imply $\left(\mathrm{A}_{n+1}\right)-\left(\mathrm{C}_{n+1}\right)$. 
Proof. First notice that for $a_{0} \in I_{n}, 0 \leqq k \leqq n$,

$$
\left|a_{k+1}\right|=\left|a_{0}+\sum_{m=0}^{k} \Delta a_{m}\right| \leqq \varepsilon^{2}+\sum_{m=0}^{k} L^{-m / 2} \varepsilon^{3} \leqq 2 \varepsilon^{2}
$$

( $\varepsilon$ small). Hence $\left(\mathrm{A}_{n}\right)$ implies that for $a_{0} \in I_{n}, g_{k+1}, k=0, \ldots, n$, satisfy the assumptions of Proposition 3.

Let us consider $\varphi^{2} \equiv \varphi_{0}^{2}+y$ with $|y|<\varepsilon$ or $|\operatorname{Im} \varphi|<L^{-1 / 2} \varrho,|\operatorname{Re} y|<L^{1 / 2} \varepsilon$.

$$
\begin{aligned}
\exp \left[-\frac{N}{2} \Delta w_{n+1}(y)\right]= & \left(\int_{-\infty}^{\infty} d s \int_{0}^{\infty} d u G_{n+1}\left(s, u, \varphi^{2}\right) / \int_{-\infty}^{\infty} d s \int_{0}^{\infty} d u G_{n}\left(s, u, \varphi^{2}\right)\right) \\
& \cdot\left(\int_{-\infty}^{\infty} d s \int_{0}^{\infty} d u G_{n}\left(s, u, \varphi_{0}^{2}\right) / \int_{-\infty}^{\infty} d s \int_{0} d u G_{n+1}\left(s, u, \varphi_{0}^{2}\right)\right) .
\end{aligned}
$$

The main contribution is, see (5.7), (5.25), (5.27), and (5.28).

$$
\begin{array}{rl}
\int_{\operatorname{Re} \mathcal{O}} & d s d u G_{n+1}\left(s, u, \varphi^{2}\right) / \int_{\operatorname{Re} \mathscr{O}} d s d u G_{n}\left(s, u, \varphi^{2}\right) \\
& =\int_{\operatorname{Re} \mathcal{O}} d s d u \exp \left[-\frac{N}{2} V_{n+1}(s, u, y)\right] / \int_{\operatorname{Re} \mathcal{O}} d s d u \exp \left[-\frac{N}{2} V_{n}(s, u, y)\right] \\
& =\exp \left[-\frac{N}{2} \int_{0}^{1} d t\left\langle\Delta V_{n}(\cdot, \cdot, y)\right\rangle\right.
\end{array}
$$

where

$$
\langle-\rangle^{t}=\int_{\operatorname{Re} \mathscr{O}} d s d u-\exp \left[-\frac{N}{2} V^{t}(s, u, y)\right] / \int_{\operatorname{Re} \mathscr{O}} d s d u \exp \left[-\frac{N}{2} V^{t}(s, u, y)\right],
$$

with $V^{t}$ given by (5.28) with $w$ replaced by

$$
w^{t}=t w_{n+1}+(1-t) w_{n} .
$$

Here $w^{t}$ satisfies (b) of Sect. 5. So we shall control $\left\langle V_{n}\right\rangle^{t}$ as in Sect. 3, changing the $u$ integration contour according to Fig. 1 [with $u_{0}(y)=u_{0}^{t}(y)$ being determined by $w^{t}$ now]. We have

$$
\left\langle\Delta V_{n}(\cdot, \cdot, y)\right\rangle^{t}=\Delta V_{n}\left(0, u_{0}^{t}(y), y\right)+\left\langle\Delta V_{n}(\cdot, \cdot, y)-\Delta V_{n}\left(0, u_{0}^{t}(y), y\right)\right\rangle^{t} .
$$

Proceeding as in Sect. 5 [see (5.33) and (5.34)], we may rewrite the second term on the right hand side of (9) as

$$
\begin{aligned}
& \left(\alpha(B-A) \int_{-1}^{1} d \sigma_{1} \int_{0}^{1} d \sigma_{2} F(\sigma) \exp \left[-\frac{N}{2}(\sigma \mid \mathscr{A} \sigma)-N U(\sigma)+O(\varepsilon)\right]\right. \\
& \left.\quad+\alpha(A-C) \int_{-1}^{1} d \sigma_{1} \int_{-1}^{0} d \sigma_{2} F(\sigma) \exp \left[-\frac{N}{2}\left(\sigma \mid \mathscr{A}^{\prime} \sigma\right)-N U^{\prime}(\sigma)+O(\varepsilon)\right]\right) / \\
& \left(\alpha(B-A) \int_{-1}^{1} d \sigma_{1} \int_{0}^{1} d \sigma_{2} \exp \left[-\frac{N}{2}(\sigma \mid \mathscr{A} \sigma)-N U(\sigma)+O(\varepsilon)\right]\right. \\
& \left.\quad+(A-C) \int_{-1}^{1} d \sigma_{1} \int_{-1}^{0} d \sigma_{2} \exp \left[-\frac{N}{2}\left(\sigma \mid \mathscr{A}^{\prime} \sigma\right)-N U^{\prime}(\sigma)+O(\varepsilon)\right]\right)
\end{aligned}
$$


where $\mathscr{A}, U$ are as before, $\mathscr{A}^{\prime}, U^{\prime}$ correspond to the $C A$ piece of the contour on Fig. 1 and

$$
|F(\sigma)| \leqq L^{-n / 2} O\left(\varepsilon^{11 / 4}\right)|\sigma|
$$

Straightforward estimation shows now that (10) is bounded by

$$
L^{-n / 2} O\left(\varepsilon^{11 / 4} N^{-1 / 2}\right)
$$

Hence

$$
\left\langle\Delta V_{n}(\cdot, \cdot, y)\right\rangle^{t}=\Delta V_{n}\left(0, u_{0}^{t}(y), y\right)+L^{-n / 2} O\left(\varepsilon^{11 / 4} N^{-1 / 2}\right) .
$$

But by (5.28), the $N=\infty$ contribution

$$
\begin{aligned}
\Delta V_{n}\left(0, u_{0}^{t}(y), y\right)= & L^{3} \Delta w_{n}\left(L^{-1} y+u_{0}^{t}(y)-1\right)=L^{3} \Delta a_{n}\left(L^{-1} y+u_{0}^{t}(y)-1\right) \\
& +\frac{1}{2} L^{3} \Delta \lambda_{n}\left(L^{-1} y+u_{0}^{t}(y)-1\right)^{2}+L^{3} \Delta \tilde{\tilde{w}}_{n}\left(L^{-1} y+u_{0}^{t}(y)-1\right) .
\end{aligned}
$$

In order to mimic (5.29) introduce

$$
\Delta w_{n+1}^{t}(y) \equiv \Delta V_{n}\left(0, u_{0}^{t}(y), y\right)-\Delta V_{n}\left(0, u_{0}^{t}(0), 0\right),
$$

and write

$$
\Delta w_{n+1}^{t}(y)=\Delta a_{n+1}^{t} y+\frac{1}{2} \Delta \lambda_{n+1}^{t} y^{2}+\Delta \tilde{\tilde{w}}_{n+1}^{t}(y),
$$

where

$$
\Delta \tilde{\tilde{w}}_{n+1}^{t}(0)=\Delta \tilde{\tilde{w}}_{n+1}^{t \prime}(0)=\Delta \tilde{\tilde{w}}_{n+1}^{t \prime \prime}(0)=0 .
$$

By (5.12) and Lemma 1,

$$
\begin{gathered}
\left|\Delta V_{n}\left(0, u_{0}^{t}(0), 0\right)\right| \leqq L^{-n / 2} O\left(\varepsilon^{5}\right), \\
\Delta a_{n+1}^{t}=L^{3} \Delta a_{n}\left(L^{-1}\left(1+L^{3} \lambda^{t}\right)^{-1}+\tilde{u}^{t \prime}(0)\right)+L^{3} \Delta \lambda_{n} \\
\cdot\left(u_{0}^{t}(0)-1\right)\left(L^{-1}\left(1+L^{3} \lambda^{t}\right)^{-1}+\tilde{u}^{t \prime}(0)\right) \\
+L^{3} \Delta \tilde{\tilde{w}}_{n}^{\prime}\left(u_{0}{ }^{t}(0)-1\right)\left(L^{-1}\left(1+L^{3} \lambda^{t}\right)^{-1}+\tilde{u}^{t \prime}(0)\right) \\
=L \Delta a_{n}+L^{-n / 2} O\left(\varepsilon^{7 / 2}\right) .
\end{gathered}
$$

Moreover

$$
\begin{aligned}
\Delta w_{n+1}^{t^{\prime \prime}}(y) & =L^{3} \Delta a_{n} \tilde{u}^{t \prime}(y)+L^{3} \Delta \lambda_{n}\left(L^{-1}\left(1+L^{3} \lambda^{t}\right)^{-1}+\tilde{u}^{t^{\prime}}(y)\right)^{2} \\
& +L^{3} \Delta \lambda_{n}\left(L^{-1} y+u_{0}^{t}(y)-1\right) \tilde{u}^{t \prime}(y)+L^{3} \Delta \tilde{\tilde{w}}_{n}^{\prime}\left(L^{-1} y+u_{0}^{t}(y)-1\right) \tilde{u}^{t \prime \prime}(y) \\
& +L^{3} \Delta \tilde{\tilde{w}}_{n}^{\prime \prime}\left(L^{-1} y+u_{0}^{t}(y)-1\right)\left(L^{-1}\left(1+L^{3} \lambda^{t}\right)^{-1}+\tilde{u}^{t^{\prime}}(y)\right)^{2} .
\end{aligned}
$$

Hence, again using (5.12) and Lemma 1, we obtain

and

$$
\Delta \lambda_{n+1}^{t}=L^{-1} \Delta \lambda_{n}+L^{-n / 2} O\left(\varepsilon^{7 / 4}\right),
$$

$$
\left|\Delta \tilde{\tilde{w}}_{n+1}^{t}(y)\right| \leqq 2 L^{-1} \sup _{|y|<\varepsilon}\left|\Delta \tilde{\tilde{w}}_{n}^{\prime \prime}(y)\right|+L^{-n / 2} O\left(\varepsilon^{3 / 2}\right) .
$$


Gathering (6), (13), (15), and (17) we obtain

$$
\begin{aligned}
& \int_{\operatorname{Re} \mathcal{O}} d s d u G_{n+1}\left(s, u, \varphi^{2}\right) / \int_{\operatorname{Re} \mathcal{O}} d s d u G_{n}\left(s, u, \varphi^{2}\right) \\
& \quad=\exp \left[-\frac{N}{2} \int_{0}^{1} d t \Delta w_{n+1}^{t}(y)+N L^{-(n+1) / 2} O\left(\varepsilon^{5}\right)\right],
\end{aligned}
$$

where $\Delta w_{n+1}^{t}$ has the properties stated in (16), (18), (20), and (21). We recall that all the time we consider $\varphi^{2}=\varphi_{0}^{2}+y$ with $|y|<\varepsilon$ or $|\operatorname{Im} \varphi|<L^{-1 / 2} \varrho,|\operatorname{Re} y|<L^{1 / 2} \varepsilon$.

In order to estimate the contribution of $\mathbb{R}^{1} \times \mathbb{R}_{+}^{1} \backslash \mathcal{O}$ to the right hand side of (5), we notice that

$$
\begin{aligned}
\int G_{n+1} / \int G_{n}= & \left(\int_{\operatorname{Re} \mathcal{O}} G_{n+1} / \int_{\operatorname{Re} \mathcal{O}} G_{n}\right)\left\{1+\left(1+\int_{\sim \operatorname{Re} \mathcal{O}} G_{n} / \int_{\operatorname{Re} \mathcal{O}} G_{n}\right)^{-1}\right. \\
& {\left.\left[\left(1-\int_{\operatorname{Re} \mathcal{O}} G_{n+1} / \int_{\operatorname{Re} \mathcal{O}} G_{n}\right) \int_{\sim \operatorname{Re} \mathcal{O}} G_{n+1} / \int_{\operatorname{Re} \mathcal{O}} G_{n+1}+\int_{\sim \operatorname{Re} \mathcal{O}} \Delta G_{n} / \int_{\operatorname{Re} \mathcal{O}} G_{n}\right]\right\} . }
\end{aligned}
$$

In Sect. 5 we have seen that

$$
\left|\int_{\sim \operatorname{Re} \mathcal{O}} G_{n+1} / \int_{\operatorname{Re} \mathscr{O}} G_{n+1}\right|<e^{-\delta N} \text { for } \delta>0 .
$$

Similarly, using $\left|e^{x}-e^{y}\right| \leqq \frac{1}{2}|x-y|\left(e^{\operatorname{Re} x}+e^{\operatorname{Re} y}\right)$ and (16)-(22), we obtain

$$
\left|\left(1-\int_{\operatorname{Re} \mathcal{O}} G_{n+1} / \int_{\operatorname{Re} \mathcal{O}} G_{n}\right) \int_{\sim \operatorname{Re} \mathcal{O}} G_{n+1} / \int_{\operatorname{Re} \mathcal{O}} G_{n+1}\right| \leqq N L^{-n / 2} O\left(\varepsilon^{11 / 4}\right) e^{-\delta N} .
$$

Notice also that $N^{-1} L^{-n / 2} \Delta G_{n}\left(s, u, \varphi^{2}\right)$ satisfies (5.46) and (5.47) as well as $G_{n}\left(s, u, \varphi^{2}\right)$. Indeed, when estimating $\Delta G_{n}$, we have to use besides (5.42) also

$$
\left|N^{-1} L^{-n / 2} \Delta g_{n}\left(\varphi^{2}\right)\right| \leqq \exp \left[-\frac{N}{12} \lambda_{\infty}(\operatorname{Re} y)^{2}+\frac{N}{2} \lambda_{\infty}(\operatorname{Im} y)^{2}+N O\left(\varepsilon^{11 / 4}\right)\right],
$$

which follows easily from $\left(\mathrm{A}_{n}\right)-\left(\mathrm{C}_{n}\right)$ for all $\varphi$ with $|\operatorname{Im} \varphi|<L^{-1 / 2} \varrho$. Proceeding as in Sect. 5, we obtain

$$
\left|\int_{\sim \operatorname{Re} \mathcal{O}} \Delta G_{n}\right| \int_{\operatorname{Re} \mathcal{O}} G_{n} \mid \leqq N L^{-n / 2} e^{-\delta N}
$$

Gathering of (22)-(25) and (27) gives

$$
\begin{gathered}
\int_{-\infty}^{\infty} d s \int_{0}^{\infty} d u G_{n+1}\left(s, u, \varphi^{2}\right) / \int_{-\infty}^{\infty} d s \int_{0}^{\infty} d u G_{n}\left(s, u, \varphi^{2}\right) \\
=\exp \left[-\frac{N}{2} \int_{0}^{1} \Delta w_{n+1}^{t}(y)+N L^{-(n+1) / 2} O\left(\varepsilon^{5}\right)\right]
\end{gathered}
$$

Thus by virtue of (5)

$$
\Delta w_{n+1}(y)=\int_{0}^{1} \Delta w_{n+1}^{t}(y)+N L^{-(n+1) / 2} O\left(\varepsilon^{5}\right)
$$


and $\left(B_{n+1}\right)$, as well as (3) for $|\operatorname{Im} \varphi|<L^{-1 / 2} \varrho,|y| \geqq \varepsilon$, and $|\operatorname{Re} y|<L^{1 / 2} \varepsilon$, follows easily from the properties (16), (18), (20), and (21) of $\Delta w_{n+1}^{t}$. We notice also that

$$
\Delta a_{n+1}=L \Delta a_{n}+L^{-(n+1) / 2} O\left(\varepsilon^{7 / 2}\right) \text {, }
$$

So that it sweeps $L^{-n / 2+1}\left[-\varepsilon^{3}+O\left(\varepsilon^{7 / 2}\right), \varepsilon^{3}-O\left(\varepsilon^{7 / 2}\right)\right]$, when $a_{0}$ runs through $I_{n}$. Hence the existence of $I_{n+1} \subset I_{n}$ with the properties stated in $\left(\mathrm{A}_{n+1}\right)$ follows.

We are left with proving (3) for $\varphi^{2}$ with $|\operatorname{Im} \varphi|<L^{-1 / 2} \varrho,|\operatorname{Re} y| \geqq L^{1 / 2} \varepsilon$. This is done the same way as the analogical part of the proof of (c) for $\tilde{g}_{1}$ in Sect. 5, by virtue of the remark that $N^{-1} L^{-n / 2} \Delta G_{n}\left(s, u, \varphi^{2}\right)$ satisfies (5.46) and (5.47).

From Proposition 4 it follows that for $a_{0} \in \cap I_{k},\left(\mathrm{~B}_{n}\right)$ and $\left(\mathrm{C}_{n}\right)$ are satisfied for each $n$ and $\left|\Delta a_{n}\right| \leqq L^{-n / 2} \varepsilon^{3}$. This implies the following result, the culmination of Sects. 4-6:

Theorem 2. Suppose that $L>L_{0}$. Given $\tilde{g}_{0}$ satisfying (a)-(c) of Sect. 5 with $\varrho<\varrho_{0}(L)$ and $N>N_{0}(L, \varrho)$, there exists $a_{0},\left|a_{0}\right| \leqq \varepsilon^{2}$, such that the iterates of $(4.1), g_{n}\left(\varphi^{2}\right)$ $=\tilde{g}_{n}\left(\varphi^{2}\right) \exp \left[-\frac{N}{2} a_{n}\left(\varphi^{2}-\varphi_{0}^{2}\right)\right]$, converge to a fixed point of $(4.1), g_{\infty}$, uniformly for $|\operatorname{Im} \varphi|<\varrho$. Moreover $\tilde{g}_{\infty}$ satisfies (a)-(c) of Sect. 5 and $\left|a_{\infty}\right| \leqq 2 \varepsilon^{2}$ (so in particular $g_{\infty}$ is non-Gaussian).

In forthcoming publications we shall try to extend our methods to the hierarchical model correlation functions and beyond the hierarchical approximation. The latter should produce, among the other things, a rigorous control of the critical point $\lambda\left(\varphi^{2}\right)^{2}$ many component quantum field theory in $d=3$. The study of the even more interesting $d=2$ case is also under way.

\section{References}

1. Amit, D. : Field theory, the renormalization group and critical phenomena. New York: McGraw Hill 1978

2. Bleher, P.M.: In multicomponent random systems (in Russian), Dobrushin, R.L., Sinai, Ya.G. (eds.). Moscow: Nauka 1978

3. Bleher, P.M., Missarov, M.D.: The equations of Wilson's renormalization group and analytic renormalization. I. General results, and II. Solution of Wilson's equations. Commun. Math. Phys. 74, 235-254 and 255-272 (1980)

4. Bleher, P.M., Sinai, Ya.G.: Investigation of the critical point in models of the type of Dyson's hierarchical models. Commun. Math. Phys. 33, 23-42 (1973)

5. Bleher, P.M., Sinai, Ya.G.: Critical indices for Dyson's asymptotically hierarchical models. Commun. Math. Phys. 45, 247-278 (1975)

6. Collet, P., Eckmann, J.-P.: The $\varepsilon$-Expansion for the hierarchical model. Commun. Math. Phys. 55, 67-96 (1977)

7. Collet, P., Eckmann, J.-P.: A renormalization group analysis of the hierarchical model in statistical mechanics. In: Lecture Notes in Physics, Vol. 74. Berlin, Heidelberg, New York: Springer 1978

8. Dyson, F.J.: Existence of a phase-transition in a one-dimensional Ising ferromagnet. Commun. Math. Phys. 12, 91-107 and Non-existence of spontaneous magnetization in a one-dimensional Ising ferromagnet. 212-215 (1969)

9. Gawȩdzki, K., Kupiainen, A.: Block spin renormalization group for dipole gas and $(\nabla \phi)^{4}$. Helsinki University preprint 1982

10. Gawȩdzki, K., Kupiainen, A.: Rigorous renormalization group and asymptotic freedom. IHES preprint 1982 
11. Kadanoff, L.P.: Scaling laws for Ising models near $T_{c}$. Physics 2, 263-272 (1966)

12. Ma, S.: Renormalization group in the large $N$ limit. Phys. Lett. 43 A, 475-476 (1973)

13. Ma, S.: Introduction to the renormalization group. Rev. Mod. Phys. 45, 589-614 (1973)

14. Ma, S.: Modern theory of critical phenomena. New York: Benjamin 1976

15. Phase transitions and critical phenomena. Domb, D., Green, M.S. (eds.), Vol. 6. New York: Academic Press 1976

16. Sinai, Ya.G.: Mathematical problems in theoretical physics. In: Lecture Notes in Physics, Vol. 80. Berlin, Heidelberg, New York: Springer 1978

17. Sinai, Ya.G.: Theory of phase transitions. Rigorous results (in Russian). Moscow: Nauka 1980

18. Wallace, D.J., Zia, R.K.P.: Rep. Prog. Phys. 41, 1-85 (1978)

19. Wilson, K.G., Fisher, M.E.: Critical exponents in 3.99 dimensions. Phys. Rev. Lett. 28, 240-243 (1972)

20. Wilson, K.G., Kogut, J.B.: The renormalization group and the $\varepsilon$ expansion. Phys. Rep. 12C, 75-199 (1974)

Communicated by A. Jaffe

Received January 7, 1983 